\title{
Metasurfaces for Stealth Applications: A Comprehensive Review
}

\author{
Vineetha Joy ${ }^{1}\left[\right.$ Alka Dileep $^{2} \cdot$ P. V. Abhilash ${ }^{1} \cdot$ Raveendranath U. Nair $^{1} \cdot$ Hema Singh $^{1}$
}

Received: 3 November 2020 / Accepted: 31 March 2021 / Published online: 23 April 2021

(c) The Minerals, Metals \& Materials Society 2021

\begin{abstract}
Metasurfaces are ultrathin, two-dimensional structures composed of periodic or quasi-periodic arrays of sub-wavelength scatterers. They possess the unique ability to comprehensively control the phase, amplitude and polarization of incident electromagnetic waves with added advantages such as ease of fabrication and less space consumption. On account of these factors, they are progressively replacing their three-dimensional counterparts, i.e. metamaterials in a wide gamut of fields such as signal multiplexing, stealth technology, holographic imaging, planar optical devices, polarization transformation devices and so on. Further, metasurfaces offer a strong and promising platform for aerospace applications due to their diversified functionalities and reduced weight penalties. Moreover, it has been widely used for the realization of thin, broadband and polarization independent radar absorbing structures (RAS). In this regard, this paper presents a concise review on the recent advancements in the field of metasurfaces specifically for stealth applications. Special emphasis has been laid on diffusion and coding metasurfaces due to their attractive properties towards the realization of low observable platforms. Furthermore, various types of metasurfaces as well as the different techniques used for the optimization of metasurfaces are also described in detail.
\end{abstract}

Keywords Metasurfaces radar cross section $\cdot$ radar absorbing structures $\cdot$ diffusion metasurface $\cdot$ coding metasurface

\section{Introduction}

The concept of metamaterial has been of immense research interest for decades due to their unusual capabilities in accomplishing electromagnetic (EM) properties not normally found in nature. They are artificially engineered structures consisting of periodically arranged unit cells and are best described by effective medium theory. ${ }^{1}$ Metamaterials evoke extraordinary EM responses such as negative-refraction index, artificial magnetism, EM-induced transparency effects, and invisibility cloaking w.r.t. incident waves. ${ }^{2}$ However, volumetric bulkiness, requirement to precisely align different layers, significant ohmic losses at optical frequencies, fabrication complexity, etc. are certain factors limiting their utility for real-world applications. In spite of the exceptional EM properties exhibited by metamaterials especially for stealth applications, ${ }^{3}$ they are progressively being

Vineetha Joy

vineethajoy@nal.res.in

1 Centre for Electromagnetics, CSIR-NAL, Bangalore 560017, India

2 Indian Institute of Technology, Kanpur 208016, India replaced by their two-dimensional counterpart, metasurfaces, due to the aforementioned barriers. ${ }^{4}$ Further, amongst other available material candidates, metal alloy compounds are used for several practical applications including that for magnetostatic shielding. However, these compounds are easily affected by changes in temperature, oxygen levels and electromagnetic radiation. ${ }^{5} \mathrm{M}$-type barium hexaferrites, with their exceptional chemical stability and resistance to corrosion, present an attractive alternative in such cases. For this reason, they are widely used in the synthesis of microwave absorbers. ${ }^{6}$ But they are inherently narrowband and exhibit increased payload due to their high density. ${ }^{7}$ Further, gallium-doped hexaferrites and nano-composite based dielectric and magnetic materials can be used to enhance absorption performance. ${ }^{8,9}$

Metasurfaces are generally composed of an array of artificially engineered sub-wavelength scattering particles, i.e., meta-atoms with uniform or non-uniform arrangement. Because of the sub-wavelength characteristics of metaatoms, metasurfaces can be considered homogenous structures with their response characterized by effective parameters. The distribution of meta-atoms on the surface can be represented by equivalent boundary conditions using electric 
and magnetic susceptibilities. This method of homogenization employing generalized sheet-transition conditions has been widely used for the development of fractal metasurfaces, leaky wave antennas, etc. Another popular technique used for modelling metasurfaces is using Huygen's principle. On striking a surface, an electromagnetic wave induces electric and magnetic dipole moments which in turn produce surface currents. In accordance with Schelkunoff's principle, these induced currents can be replaced with equivalent tangential electric and magnetic fields which tailors the electromagnetic response of the metasurface. Therefore, each individual sub-wavelength element must be polarizable for a surface to be characterized as a metasurface. ${ }^{10}$

Unlike the bulky three-dimensional metamaterials, metasurfaces are ultra-thin structures with negligible thickness and hence occupy less physical space. The thin profile of metasurfaces, designed using suitable materials and meta-atoms, also aids in reducing undesirable losses in the direction of wave propagation. Moreover, they provide more degrees of freedom to accomplish spatial inhomogeneity over a thin surface. In comparison with the application of frequency selective surfaces (FSS) for radar cross-section (RCS) reduction, the dimensions, positions and spatial arrangement of constituent sub-wavelength meta atoms of metasurfaces have more flexibility in accomplishing the same. Furthermore, the fabrication of metasurfaces is much easier than that of metamaterials. A notable difference exists in the methodology of characterization as well. While metamaterials are described by effective medium theory, generalized sheet transition conditions are adopted for modelling metasurfaces due to their thin profile. ${ }^{11}$ The diversified EM manipulation capabilities of metasurfaces in combination with their reduced weight penalties make them excellent choices for accomplishing stealth characteristics on various military platforms. In this regard, the present paper gives a concise review on the recent advancements in the field of metasurfaces for low observable applications. Special emphasis has been laid on diffusion and coding metasurfaces due to their attractive properties like broad bandwidth, polarization insensitivity and reduced thickness. Furthermore, various types of metasurfaces as well as the different techniques used for the optimization of metasurfaces are described in detail.

\section{Overview of Metasurfaces}

Metasurfaces are basically planar metamaterial structures that control the phase, amplitude, frequency, angular momentum and polarization of EM waves by intelligent manipulation of boundary conditions. ${ }^{12}$ When an electromagnetic wave is incident on a static but spatially inhomogeneous metasurface, the frequency remains unchanged. However, the transverse momentum varies due to the change in momentum caused by the metasurface. This kind of spatially modulated metasurface can be used to manipulate the spatial distribution of fields. On the other hand, if the wave is incident on a dynamic (time-varying surface susceptibility) but spatially homogeneous metasurface, the frequency changes with an unchanged transverse momentum. The latter category, popularly known as time-varying metasurfaces, can be conveniently used for frequency conversion applications. In this case, the effective parameters can be made to change with time providing the impression that it is moving in a particular direction with a specific velocity. Finally, space-time-modulated metasurfaces with spatio-temporal variation of effective parameters provide options for modulating both frequency as well as momentum. This concept has been employed in Doppler cloaks for restoring the invisibility of dynamic cloaked objects. ${ }^{13-17}$

Further, the planar configuration of metasurfaces enables easy implementation at terahertz (THz) and visible spectra using standard photolithography. These extra-ordinary properties and capabilities exhibited by metasurfaces ${ }^{18}$ have led to its application in a wide gamut of fields such as signal multiplexing, stealth technology, holographic imaging, planar optical devices, and polarization transformation devices. A brief overview on the theoretical background of metasurfaces including the various types and functionalities is presented in the following sections.

\section{Theoretical Background}

When an EM wave is incident on the interface between two homogenous media with different refractive indices, the wave splits into reflected and transmitted waves as per the Snell's law of refraction. ${ }^{19}$ However, the reflection/transmission properties will change if a metasurface composed of sub-wavelength resonators is introduced at the interface. This is due to the resonant behavior of the structure which produces an effective current within the metasurface leading to phase alteration. Generalized laws of reflection and refraction have been introduced to interpret this phase discontinuity. ${ }^{20}$

The generalized laws are based on Fermat's principle which states that light always travels along the path which takes the least amount of time to traverse between two points. ${ }^{10}$ Consider a plane wave incident on a metasurface placed at the interface between Medium\#1 and Medium\#2 with refractive indices $n_{1}$ and $n_{2}$, respectively (Fig. 1). The angle of incidence and refraction are denoted by $\theta_{1}$ and $\theta_{2}$, respectively. The possible paths that the wave can take are highlighted in blue and red lines in Fig. 1. Because of the abrupt discontinuity in phase caused by the metasurface, the phase at points $P$ and $P^{\prime}$, separated by a distance $\mathrm{d} x$, are taken as $\phi$ and $\phi+\mathrm{d} \phi$, respectively. If the two paths are infinitesimally close, the phase difference between them will 


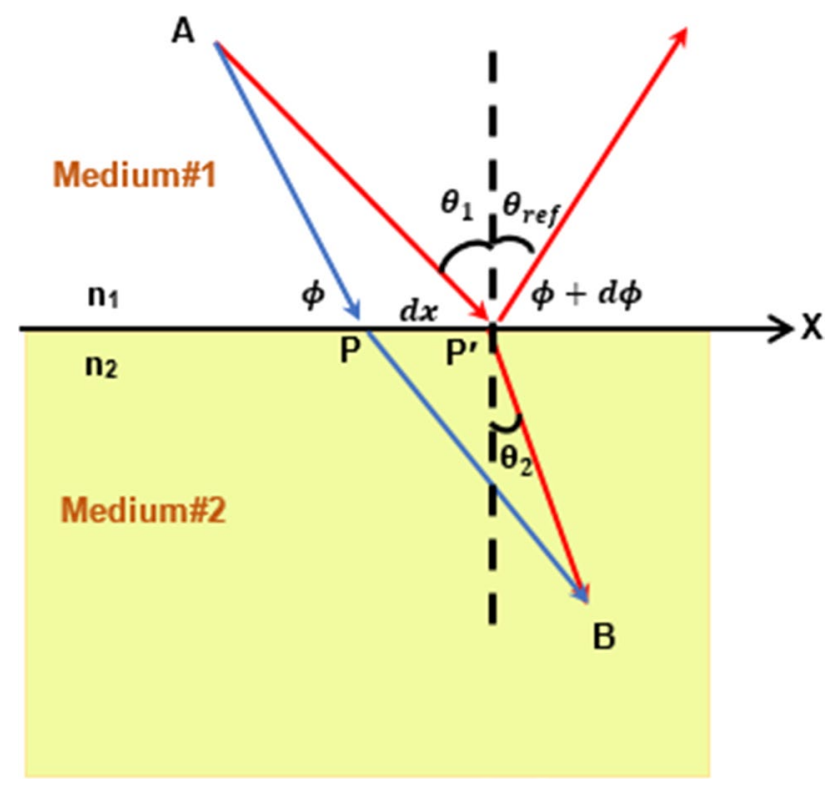

Fig. 1 EM wave propagation at the interface between two media separated by a metasurface.

be zero at the boundary. Under this assumption, the generalized Snell's law of refraction for a constant phase gradient along the interface gives, ${ }^{19}$

$n_{2} \sin \theta_{2}-n_{1} \sin \theta_{1}=\frac{\lambda_{0}}{2 \pi} \frac{\mathrm{d} \phi}{\mathrm{d} x}$

Similarly, the generalized law of reflection can be written as,

$\sin \theta_{\text {ref }}-\sin \theta_{1}=\frac{\lambda_{0}}{2 \pi n_{1}} \frac{\mathrm{d} \phi}{\mathrm{d} x}$

where $\lambda_{0}$ is the free space wavelength and $\theta_{\text {ref }}$ is the angle of reflection.

Moreover, the generalized law of reflection also confirms an angle of incidence above which the reflected wave becomes evanescent. It can be expressed as, ${ }^{19}$

$\theta_{c}=\sin ^{-1}\left(1-\frac{\lambda}{2 \pi n_{1}}\left|\frac{\mathrm{d} \phi}{\mathrm{d} x}\right|\right)$

Equation 1 shows that the refracted wave can be redirected in a desired direction by controlling the phase gradient. Further, it is clear from Eq. 2 that the angle of reflection is no longer equal to the angle of incidence for a metasurface. However, if there is no phase discontinuity at the interface $(\mathrm{d} \phi / \mathrm{d} x=0)$, i.e., in the absence of metasurface, Eqs. 1 and 2 simplify to the original Snell's law of refraction and reflection, respectively. Therefore, it is apparent that the direction of wave propagation and shape of the wavefront can be adjusted by controlling the spatial phase variation on a metasurface.

\section{Comparison Between Metasurfaces and Frequency Selective Surfaces (FSS)}

The difference between metasurfaces and FSS can be better understood by drawing an analogy to their 3D counterparts, i.e., metamaterials and conventional electromagnetic band gap (EBG) structures. ${ }^{21}$ In general, the EM behaviour of periodic 3D materials can be divided into three domains based on the operating frequency range, as shown in Fig. 2. Domain\#1 corresponds to the low frequency region, where the wavelength is much higher than the periodicity of the constituent scatterers making up the composite material. Similar to atoms/molecules in normal materials, these scatterers can have induced or permanent dipole moments. Conventional mixing formulas are used in this domain to compute the effective constitutive parameters. Moving further up along the frequency axis, there exists domain\#2 where the wavelength is still higher than the periodicity. However, the constituent scatterers can resonate by themselves in this region. The materials belonging to this domain where the scatterers resonate but not the lattice is referred to as metamaterials. Although the medium is dispersive here, they can still be represented using their effective permittivity and permeability. In domain\#3, the operating wavelength is closer to or smaller than the periodicity and consequently the EM waves will be unable to view the material as an effective medium. Full wave approaches such as Floquet-Bloch mode expansion are generally used for studying the behaviour of fields in this domain. The composite materials in this domain where lattice/array dispersion predominates, are referred to as EBG or photonic band gap (PBG) structures. These structures can have pass bands or stop bands based on the frequency of incident wave. Bragg scattering, the foundation for various practical applications, is observed in this domain. These three domains of 3D-composite materials can be correlated to the $2 \mathrm{D}$ arrangement of scatterers. In this case, domain\# 1 corresponds to conventional thin film media and lattice dispersion (resonances related to the periodicity) predominates in domain\#3. ${ }^{21}$ Frequency selective surfaces (FSS) belong to domain\#3. On the other hand, metasurfaces belong to domain\#2 where individual scatterers resonate but not the lattice. In this case, the constituent meta atoms/scatterers have to be appropriately designed so that their resonant frequency is much lower than the frequency of the next higher-order Floquet-Bloch mode. Otherwise, the material will behave as a conventional FSS. Unlike metasurfaces, FSS have limited signal processing capability. $^{22}$ 


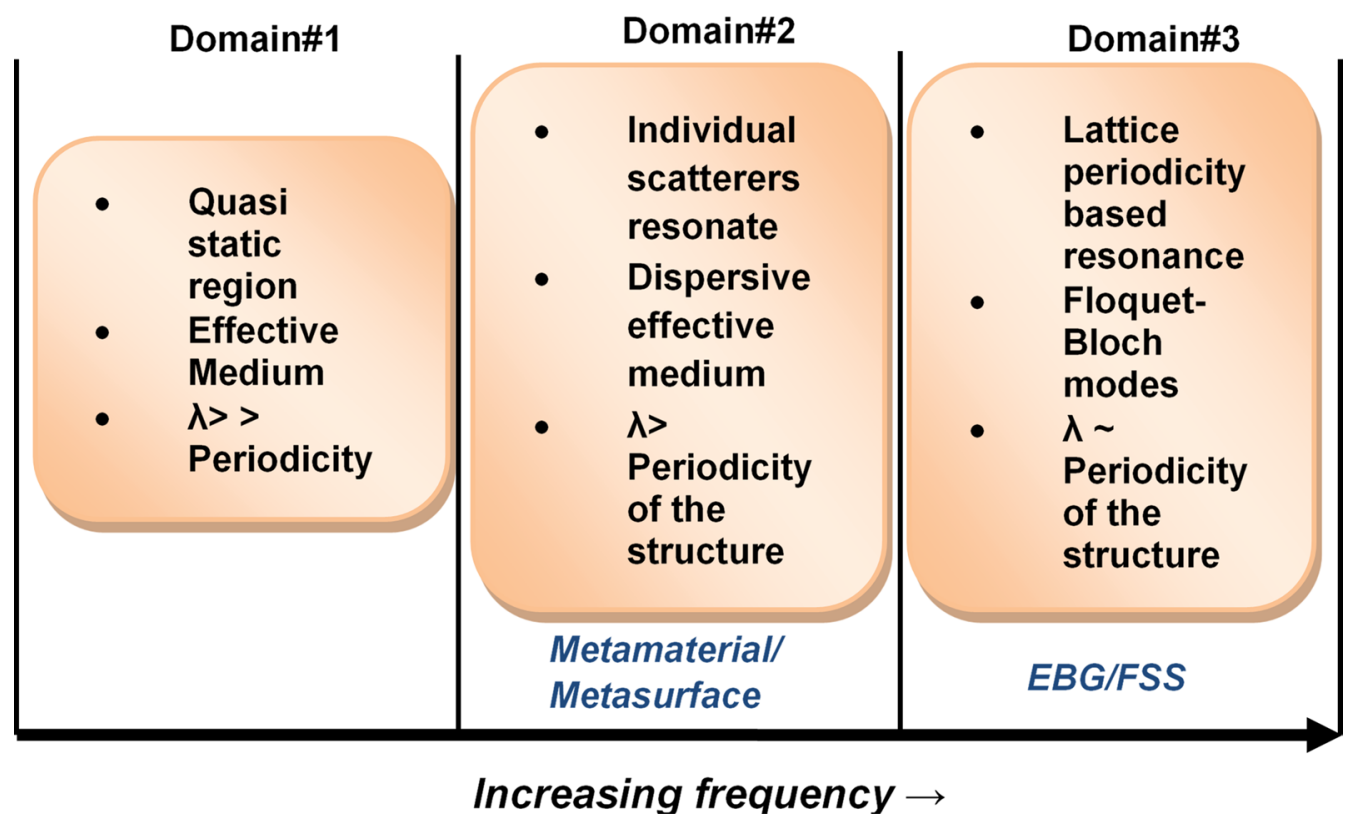

Fig. 2 Distinct domains of a composite material.

\section{Classification of Metasurfaces}

Any two-dimensional periodic structure with thickness and periodicity comparable to the operating wavelength can be considered as a metasurface. It can be broadly classified into two classes, namely metafilms and metascreens. Metasurfaces characterized by cermet configuration (array of isolated scatterers) are known as metafilms. On the other hand, metasurfaces having fishnet configuration (periodically spaced apertures) are classified as metascreens. Metasurfaces can also be categorized based on the underlying mechanism used for phase manipulation ${ }^{23}$ as follows.

\section{Multi-resonance Metasurfaces}

Multi-resonant metasurfaces are capable of producing multiple narrow resonances which can be tapped for applications such as nonlinear optics, filtering, and molecular fingerprinting. A ' $\mathrm{V}$ ' shaped plasmonic antenna with two equally long nanorods hinged at a certain angle was the first proposed structure of this kind. ${ }^{19,24}$ It supports both symmetric and anti-symmetric modes which contributes to the dual resonance characteristics, as shown in Fig. 3. These modes are excited when the polarization of incident wave is along $\bar{a}$ or $\vec{s}$ directions and if it is so, the scattered waves will have the same polarization as that of the incident wave. At oblique incidence, both the modes will get excited simultaneously, resulting in scattered waves with different amplitude and polarization. In Fig. 4, eight unit cells with the same amplitude but with a phase increment of $\pi / 4$ at cross-polarized radiation are shown. This type of metasurfaces have low efficiency due to the co-existence of both anomalous as well as ordinary reflection/refraction.

\section{Gap-Surface Plasmon Metasurfaces (GSPM)}

These are reflective array metasurfaces constructed by inserting an optically thin dielectric layer between a metallic antenna array and the ground plane. ${ }^{25}$ Here, the thin spacer leads to strong near field coupling between the antenna array and their mirror elements in the ground plane which in turn leads to phase modulation in the range of $2 \pi$. The anti-parallel currents induced on the array and the metallic ground plane generate a strong magnetic field called gap-surface plasmon modes inside the dielectric layer. In this approach, the polarization of the reflected wave will be the same as that of the incident wave and the phase shift can be effectively controlled by changing the element length. ${ }^{23}$

These metasurfaces are used in various applications such as beam steering, planar metalens design, GSPM-based hologram, ultra-thin absorber design, high resolution colour printing, polarimeter control, and detection/development of surface wave couplers. ${ }^{25}$ Poers et al. ${ }^{26}$ described the operation of gap-plasmon gradient metasurface at optical regime and experimentally proved that the two degrees of freedom of a gold nanobrick geometry can be efficiently used for the independent control of reflection phases of orthogonally polarized components. The corresponding unit cell with a gold nanobrick and a thin golden film (ground plane) separated by glass is shown in Fig. 5. The supercell of a metasurface generated using this unit cell with $80 \%$ reflectivity is shown in Fig. 6. 


\section{Symmetric Mode $\quad$ Antisymmetric Mode}
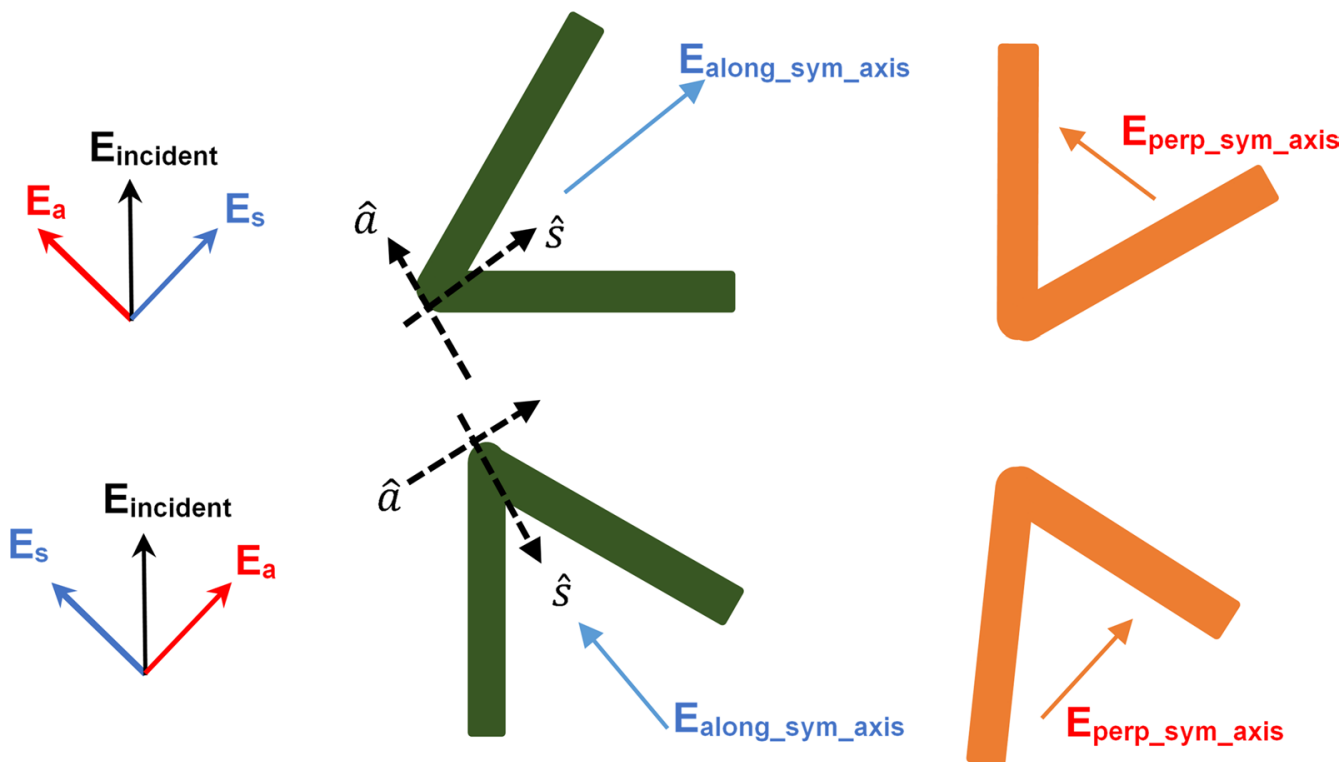

Fig. 3 'V' shaped antenna element with two resonant modes (Data from 19).
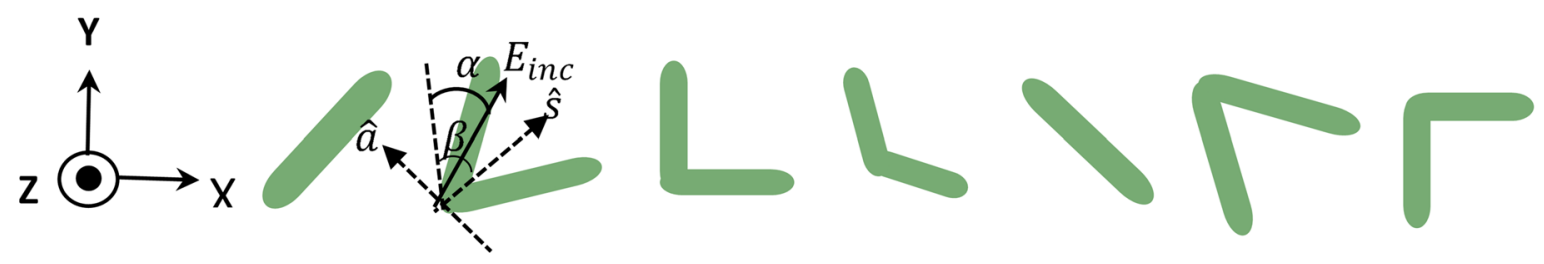

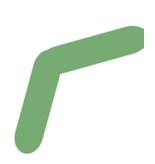

Fig. 4 Plasmonic antenna array (Data from 24).
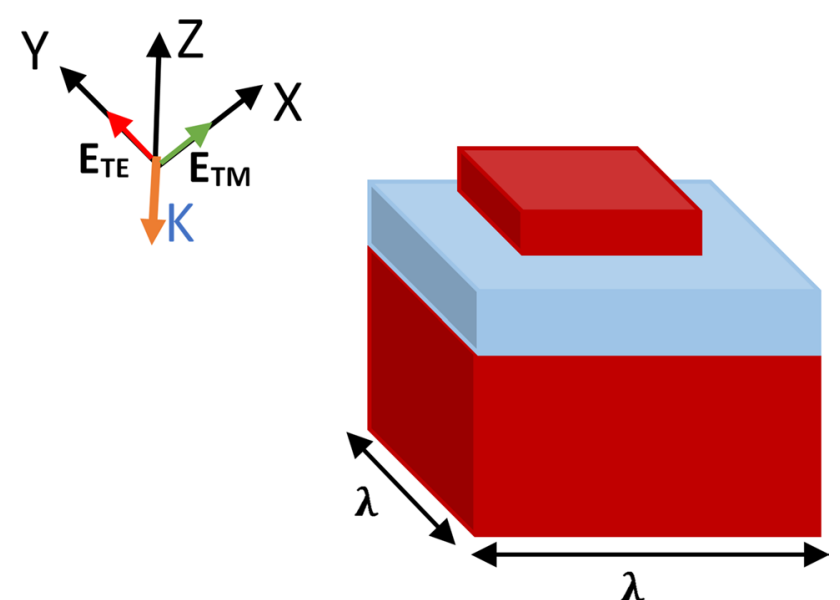

Fig. 5 Basic meta-atom of an optical GSPM with gold brick and a golden film separated by a glass spacer (Data from 25).

\section{Pancharatnam-Berry-Phase Metasurfaces}

Pancharatnam-Berry (PB) phase metasurfaces achieve phase modulation by changing the orientation angle of the unit cell. These metasurfaces are easy to design and they have large fabrication tolerance, but are limited to circularly polarised light operations. Consider an anisotropic GSP meta-atom, rotated by an angle $\theta$ from the $x$-axis, illuminated by a linearly polarized wave. The corresponding Jone's matrix can be written as,

$M_{\theta}=R(-\theta)\left(\begin{array}{cc}M_{x x} & 0 \\ 0 & M_{y y}\end{array}\right) R(\theta)$

where

$R(\theta)=\left(\begin{array}{cc}\cos \theta & \sin \theta \\ -\sin \theta & \cos \theta\end{array}\right)$

$M_{x x}$ and $M_{y y}$ represent the reflection and transmission coefficients for linearly polarized light along the two primary 


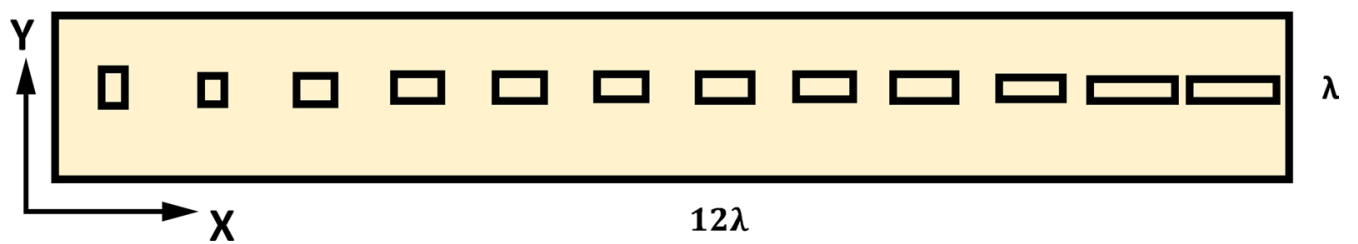

Fig. 6 Super cell of a GSPM metasurface with gold nanobricks and glass spacer (Data from 26).

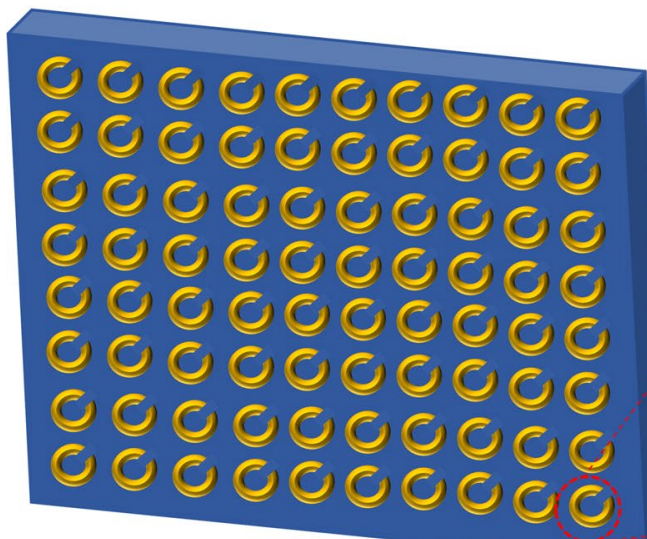

(a)

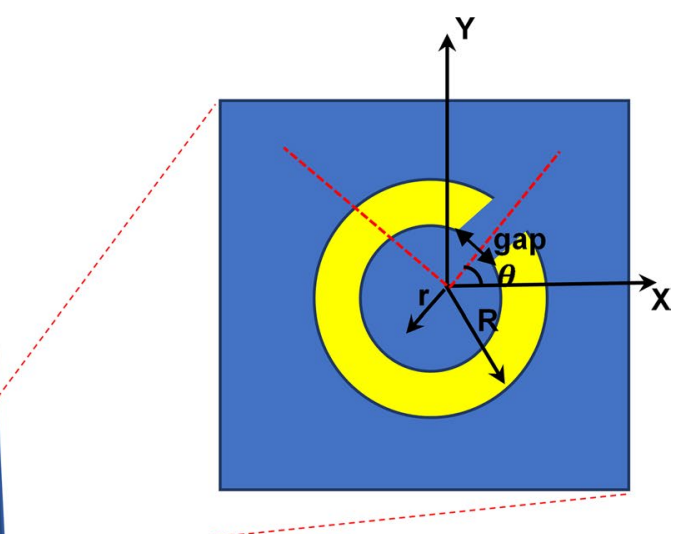

(b)

Fig. 7 Illustration of a PB phase metasurface (Data from 27) used as a beam splitter (a) metasurface (b) unit cell.

axes of the meta-atom, respectively. ${ }^{25}$ When the incident wave is circularly polarised, the reflected/transmitted field can be expressed as,

$M_{\theta} \cdot E_{i}^{ \pm}=\frac{1}{2}\left(M_{x x}+M_{y y}\right) E_{i}^{ \pm}+\frac{1}{2}\left(M_{x x}-M_{y y}\right) e^{ \pm 2 \theta} E_{i}^{\mp}$

where $E_{i}^{ \pm}$represents the incident left-handed circularly polarised light (LHCP) and right-handed circularly polarised light (RHCP), respectively. Equation 6 can be viewed as a sum of two components in which the first term is the circular polarized scattered wave with the same helicity as that of the incident wave. On the other hand, the second term indicates light with opposite helicity with an additional PB phase $( \pm 2 \theta)$. Therefore, the phase shift can be tuned from 0 to $2 \pi$ by adjusting the orientation of the meta-atoms from 0 to $\pi$.

Using PB phase technique, metasurfaces with large conversion efficiency ( $>25 \%$ ) and broad bandwidth has been developed in Ref. 27 using a ' $C$ ' shaped split-ring resonator (SRR) unit cell as shown in Fig. 7.

\section{Huygens' Metasurface}

Huygens' metasurfaces (HMS) are two-dimensional arrays of polarizable particles on a thin dielectric layer that can tailor electric as well as magnetic polarization currents to

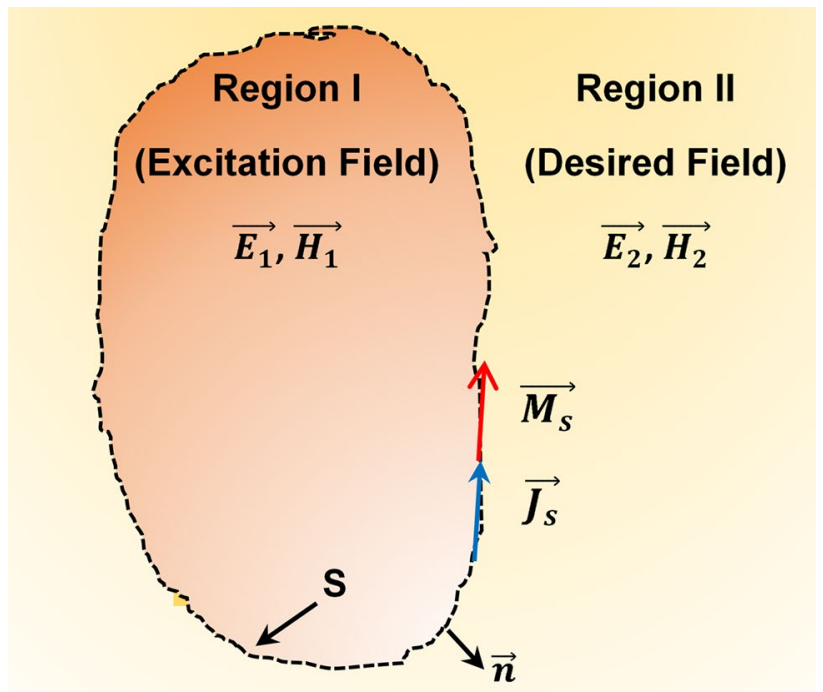

Fig. 8 Illustration of equivalence principle (arbitrary fields in two regions separated by a closed surface ' $\mathrm{S}$ ').

produce specific wave fronts. ${ }^{28}$ They are extensively used in various electromagnetic applications such as stealth technologies, polarization control devices, and beam transformers. HMS work in accordance with equivalence principle where equivalent electric $\left(J_{s}\right)$ and magnetic currents 
$\left(\vec{M}_{s}\right)$ are introduced on the interface between two media as shown in Fig. 8 to satisfy the boundary conditions.

The equivalent surface currents can be expressed as,

$\overrightarrow{J_{s}}=\hat{n} \times\left(\vec{H}_{2}-\vec{H}_{1}\right) ; \quad \vec{M}_{s}=-\hat{n} \times\left(\vec{E}_{2}-\vec{E}_{1}\right)$

These fictitious surface currents can be replaced with polarization currents that are induced when a field is incident on the array of polarizable particles. The boundary conditions shown in Eq. 7 can be transformed into impedance boundary conditions in terms of electric sheet admittance $(Y)$ and magnetic sheet impedance $(Z)$. Once the boundary condition is formulated for obtaining a specific wavefront, the suitable values of $Y$ and $Z$ can be found. Further, this required surface impedance can be implemented by subwavelength texturing of the metallic patch on a dielectric layer. $Y$ and $\mathrm{Z}$ can be conveniently defined in terms of surface polarizabilities which can also be used to represent the equivalent electric and magnetic surface currents. For a plane wave incident normally on a periodic metasurface, they can also be extracted from the complex reflection (RC) and transmission (TC) coefficients as follows:

$Y=\frac{2(1-T C-R C)}{\eta(1+T C+R C)^{\prime}} ; \quad Z=\frac{2 \eta(1-T C+R C)}{(1+T C-R C)}$

where $\eta=\sqrt{\mu / \varepsilon}$ is the free space wave impedance. It is evident from Eq. 8 that the magnitude of the meta atom's transmission coefficient tends to unity when the normalized $Y$ and $Z$ are purely imaginary and equal. Moreover, the magnitude of impedance can be modified appropriately to vary the phase of the meta-atom anywhere between $-\pi$ to $+\pi$.

Huygens' metasurfaces are extensively used in microwave as well as optical regime as they can fully control both copolarized as well as cross-polarized waves with zero reflection losses. An HMS with $86 \%$ transmission efficiency has been proposed in Ref. 28 that can refract incident waves at $45^{\circ}$ from the normal without any reflection. A broadband HMS based on mutually shifted split ring resonator (SRR) with $360^{\circ}$ phase coverage has been presented in Ref. 29. A lossless multi-layered augmented HMS has been proposed in Ref. 30 where baffles have been used in between adjacent microcells to shield one microcell from another.

\section{All-Dielectric Huygens' Metasurfaces}

Conventional metasurfaces with metallic patterns suffer from high dissipative losses in the visible frequency range. Dielectric metasurfaces have been introduced to overcome this challenge where displacement currents generate the strong optical response rather than ohmic currents. ${ }^{31}$ An all dielectric bi-anisotroptic nanoparticle based metasurface

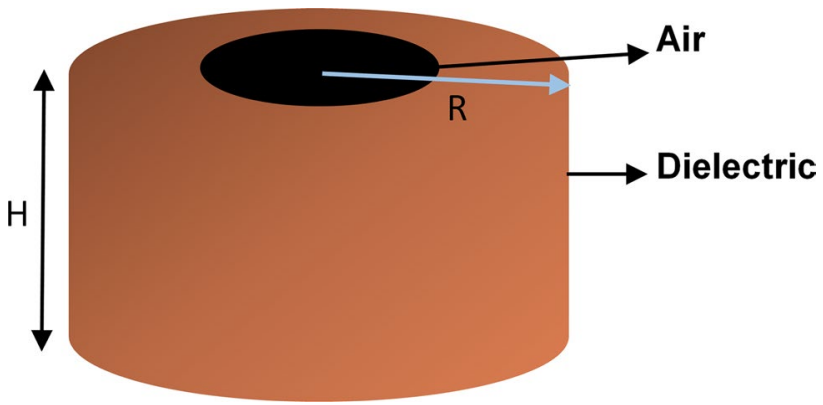

Fig. 9 Single bi-anisotropic nano-particle (Data from 33).

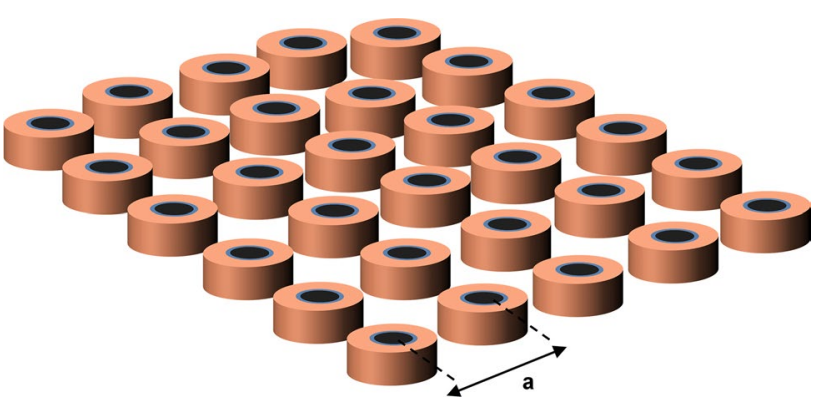

Fig. 10 All-dielectric bi-anisotropic metasurface (Data from 33).

without a metallic backing has been presented in Ref. 32 for achieving out of band transmission in reflect arrays. Here, the geometric parameters of the nanoparticle have been tuned to modify the phase.

Odit et al. ${ }^{33,34}$ experimentally realized an all-dielectric bianisotropic metasurface in the frequency range of 4-9 GHz. The measurements revealed that the excitation of metasurface from opposite directions result in different reflection phases. Moreover, a $360^{\circ}$ phase change with the amplitude of reflection coefficient close to unity has been observed at $6.8 \mathrm{GHz}$. The corresponding meta atom and metasurface are shown in Figs. 9 and 10, respectively.

\section{High-Contrast Metasurfaces}

High-contrast metasurfaces consist of periodically arranged high refractive index dielectric particles separated/disconnected from each other. These kinds of structures can control phase as well as polarization at the same time. Because of the huge difference between the refractive index of the dielectric scatterers and the surrounding environment, optical energy is predominantly focused inside these scatterers. Therefore, the transmission characteristics of the metasurface is determined by the geometry of the nanoscatterer. Under this category, visible light metasurfaces with beam steering ability and focusing property have been proposed. ${ }^{35}$ High-contrast gratings (HCG) based on gallium nitride have 
been used here to attain near unity reflection, beam steering and polarisation splitting. A flexible high contrast metasurface has been presented in Ref. 36 to modify the optical properties of any arbitrarily shaped object onto which it conforms.

Based on the remarkable properties exhibited by different classes of metasurfaces, they are extensively used in numerous applications ${ }^{37-39}$ such as polarization conversion, focusing of reflected and transmitted waves, generation of holographic images, realization of band pass/bandstop characteristics, implementation of flat lenses, and stealth technologies.

\section{Optimization of Metasurfaces}

Optimization is a crucial step in the design of highly functional metasurfaces catering to various applications. Conventionally, parameter sweep is the initial step taken by designers towards optimization. However, they are highly inefficient in dealing with multi-dimensional and multiobjective optimisation problems with several constraints imposed on input parameters. Therefore, selection of a suitable optimization method is an integral part in the design process of metasurfaces and an appropriate algorithm tailored to the problem at hand will greatly reduce the computational cost.

The selection of optimisation technique for a particular application is based on several factors like number of input parameters, input space topology, number of objectives and computational cost. ${ }^{40}$ The primary decisive factor is with respect to the discreteness of input space. The input space is termed as discrete if there are fixed number of solutions from which an optimal one is to be chosen. In such cases, genetic algorithm (GA) is preferred if the metasurface can have non-contiguous scatterers. Moreover, GA is highly efficient in searching in large solution spaces. ${ }^{41}$ Contiguous metasurfaces are those containing spatially continuous patterns such as meander lines and sinusoidally modulated metallic patches, whereas non-contiguous metasurfaces consist of discrete meta atoms. Although GA has been successfully used for the optimization of pixelated metasurfaces, its application is usually constrained to planar configurations due to the probability of disconnected pixels in conformal models. On the other hand, ant colony optimization (ACO) or multi-objective lazy ant colony optimization (MOLACO) is capable of mapping the optimal path traced by the artificial ants in the graph topology to a contiguous model. Therefore, If the metasurface is to have a contiguous pattern like in the case of meander-line antennas, ACO or MOLACO would be a better choice. ${ }^{40}$

In case of a continuous solution space, i.e., where the input parameters can take any value satisfying certain pre-defined constraints, the selection of suitable optimization algorithm is a herculean task. A local optimization technique is the best option in such scenarios provided a good initial solution exists. Further, if the problem can be framed as a finite element (FE) one, then topology optimization can be efficiently employed else gradient based approaches such as Newton's method (NM) and multi-objective gradient descent algorithm (MDGA) can be used. However, local optimization algorithms suffer from the issue of multiple local minima during the optimization process. Several strategies such as radically modifying the error function, temporarily over-designing the system, and movement from one local minimum to a neighbouring one by tracing a saddle point between them can be employed to alleviate this problem. ${ }^{42}$

In the absence of a good starting point, one may have to go for global optimization algorithms such as GA and particle swarm optimization (PSO) ${ }^{43-45}$ PSO has several advantages in comparison with GA. The population (swarm of bees) in PSO is observed to function more independently and in co-operation with other members. Moreover, in several cases like that of design of negative index metamaterials, ${ }^{46}$ PSO has been found to outperform GA. But a major disadvantage of global optimization algorithms such as PSO and GA is their sensitivity to internal parameters (size of population, acceleration constants, etc.) which must be tuned based on the problem at hand. In most cases, adaptive tuning of these parameters are required during the optimization process in order to increase the efficiency of the algorithm. Covariance matrix adaptation evolution strategy (CMA-ES) is an attractive alternative to GA and PSO w.r.t. this aspect. CMA-ES has very few user-defined internal parameters and has been found to be better than $\mathrm{GA}^{47}$ in the optimization of Bézier metasurfaces. If the time required for the evaluation of a single function is very large, then techniques such as multi-objective with tolerance (MOTOL), deep learning, and surrogate modelling must be used. Figure 11 summarizes the criteria for selecting the appropriate optimization technique to solve a task at hand.

\section{Metasurfaces for Stealth Applications}

Metasurfaces have gained significant research attention in the recent decades due to their exceptional properties coupled with simplified fabrication process and reduced weight penalties. Moreover, they are promising substitutes to their conventional three dimensional counterparts, i.e., metamaterials with respect to functionality as well as compactness. These attributes are highly beneficial for aerospace applications where a slight increase in payload can have detrimental effects on the aerodynamic performance. In this direction, the present section discusses the recent advancements in the 


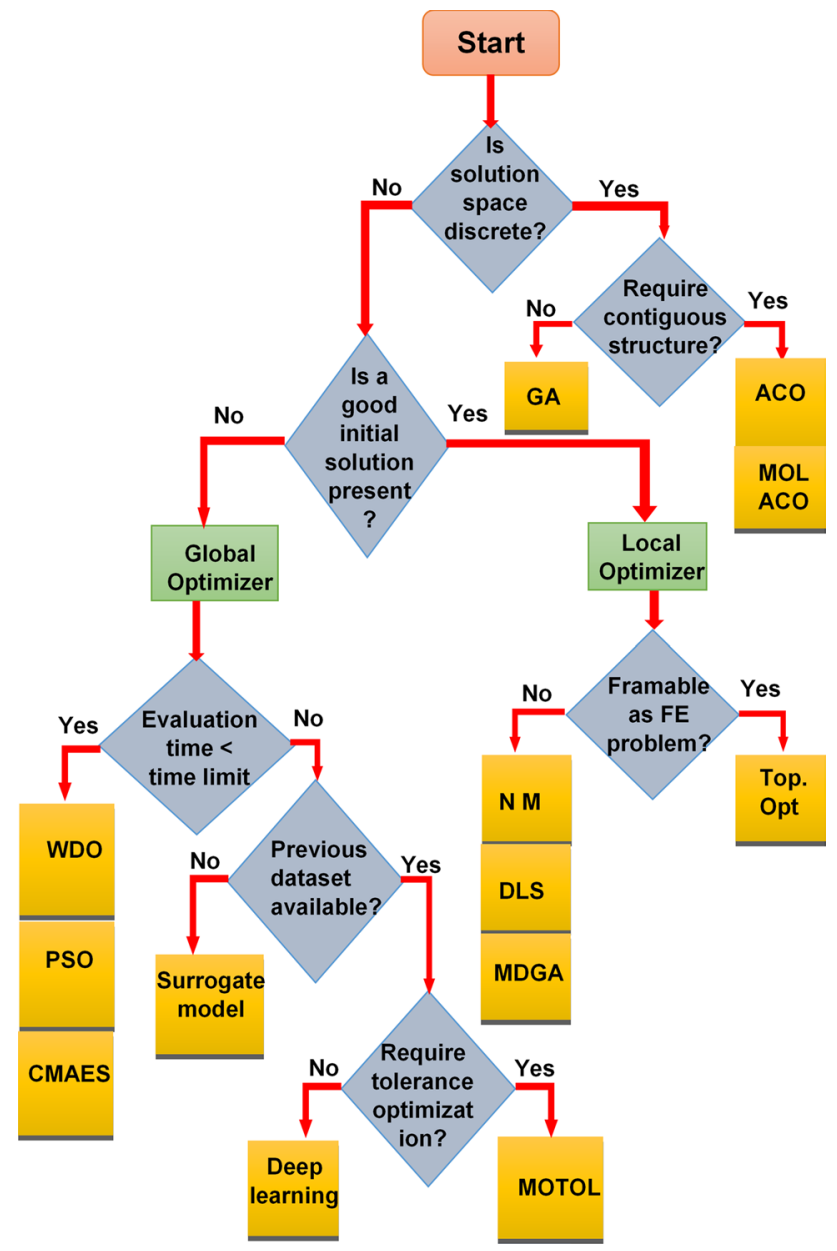

Fig. 11 Summary of the criteria used for selecting appropriate optimization techniques application of metasurfaces for aerospace applications with detailed attention to stealth technology.

\section{Metasurfaces for Radar Cross Section Reduction (RCSR)}

The capability of metasurfaces in tailoring reflected wavefronts can be effectively tapped for reducing the radar crosssection (RCS) of potential hotspots. A combination of two unit cells with closely spaced absorption peaks has been used in Ref. 48 for widening the bandwidth of operation. The combined unit cell (substrate: FR-4; thickness $=1 \mathrm{~mm}$ ) and the corresponding equivalent circuit model derived w.r.t. the direction of current flow on the unit cell is shown in Fig. 12. Here, $L_{\mathrm{I}}\left(/ L_{\mathrm{O}}\right)$ and $C_{\mathrm{I}}\left(/ C_{\mathrm{O}}\right)$ corresponds to the effective inductance and capacitance of the inner (/outer) set (complete ring and split ring), respectively. Further, $C_{\mathrm{c}}$ and $C_{\mathrm{d}}$ denote the coupling capacitance between the inner and outer sets and the effective capacitance between the top patch and the bottom metallic ground plane, respectively.

The equivalent impedance $(Z(\omega))$ of the circuit can be formulated using principles of network analysis and the absorption frequencies can be solved by imposing impedance matching conditions. By appropriately tuning the circuit parameters, the absorption frequencies can be brought closer to each other for enhanced bandwidth. The proposed structure achieved a bandwidth of $1.15 \mathrm{GHz}(9.40-10.55 \mathrm{GHz})$ with two absorption peaks at $9.66 \mathrm{GHz}$ and $10.26 \mathrm{GHz}$, respectively (range of incident angles: $0^{\circ}-40^{\circ}$ ). In a similar manner, equivalent circuits can be modelled for any unit cell for gaining better insight of the absorption mechanism.

A periodic array of quasi-L-shaped patches imprinted on a substrate and connected to the ground plane using nonsymmetric vias (Fig. 13a) has been proposed as an ultrawideband polarization rotation reflective surface (PRRS)

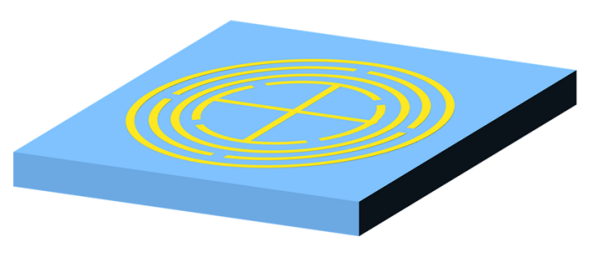

(a)

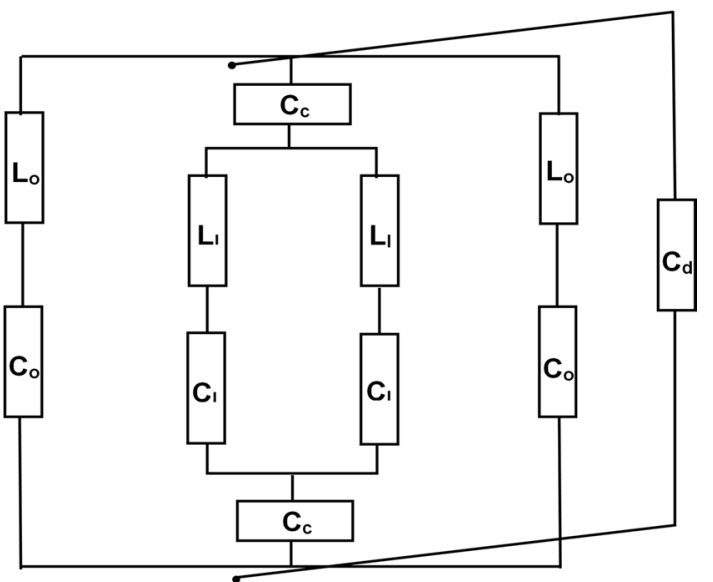

(b)

Fig. 12 Equivalent circuit modelling of meta atoms (Data from 48) (a) Unit cell, (b) Equivalent circuit. 


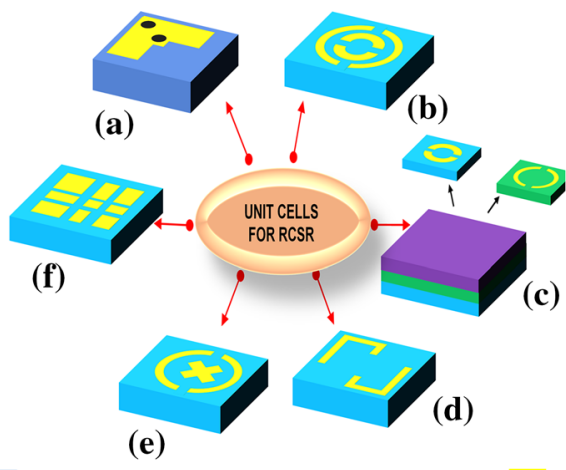

FR4 F4B

Modified_epoxy

Arlon AD4530

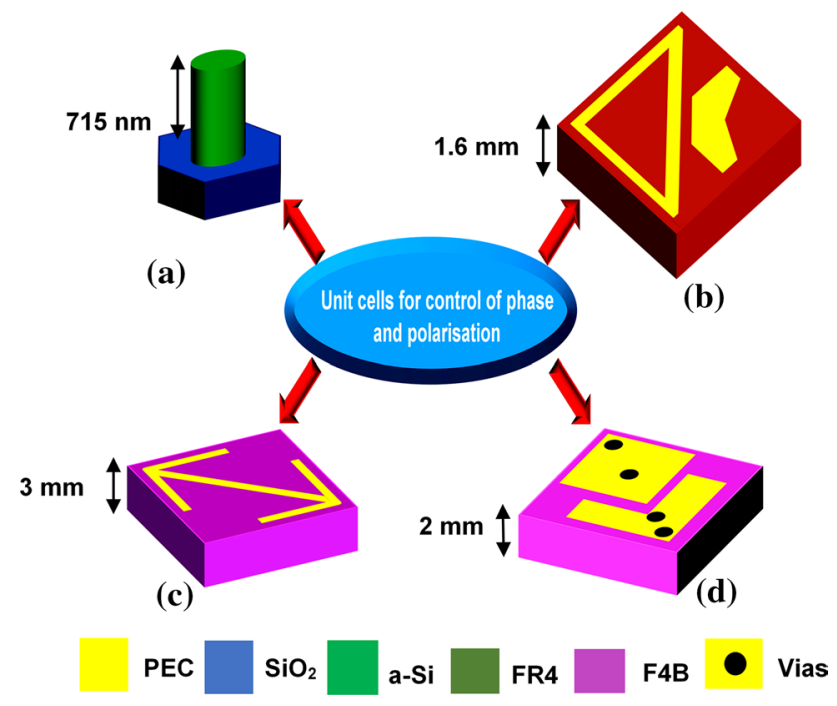

Fig. 14 Unit cells used in metasurfaces for control of phase and polarization (a) Data from 57 (b) Data from 58 (c) Data from 59 (d) Data from 60.

\section{Metasurfaces for Controlling Phase and Polarization}

The configuration and arrangement of meta-atoms on a metasurface can be efficiently optimized in order to achieve specific phase control and polarization conversion. A high contrast optical metasurface with dimensionally different elliptical dielectric nano-posts (Fig.14a) has been found to exhibit complete control over polarization and phase with a measured efficiency in the range of $72-97 \% .^{57}$ An adaptability of this nature can be used for the implementation of various optical elements such as lenses, beam splitters, polarizers, and polarization-switchable phase holograms. Khan et al. ${ }^{58}$ presented a multi-functional ultra-thin metasurface with capabilities for linear to circular polarization conversion and cross-polarization conversion in the X-band. Here, cross-polarization conversion has been accomplished over $8 \mathrm{GHz}$ to $11 \mathrm{GHz}$ with an efficiency of $95 \%$ and linear-to-circular polarization conversion has been implemented in two frequency bands from $7.5 \mathrm{GHz}$ to $7.7 \mathrm{GHz}$ and $11.5 \mathrm{GHz}$ to $11.9 \mathrm{GHz}$. The unit cell, shown in Fig. 14b has also been found to be stable in its performance over a wide range of incident angles for both TE as well as TM polarizations. Chen et al. 59 experimentally realized an ultra-wideband metasurface based on a double-head arrow structure (Fig. 14c) with nearly $100 \%$ polarization conversion efficiency at $6.8 \mathrm{GHz}, 12.17 \mathrm{GHz}$, $15.45 \mathrm{GHz}$, and $23.13 \mathrm{GHz}$. Further, a polarization rotation reflective surface (PRRS), shown in Fig. 14d has been used for achieving 10-dB RCS reduction from $6.1 \mathrm{GHz}$ to $17.8 \mathrm{GHz}^{60}$ 


\section{Diffusion Metasurfaces for RCSR}

A conducting patterned surface with uniform distribution of phase orthogonal to the direction of radiation is conventionally used for focussing EM energy in the specular direction. In the case of conducting surfaces with uniform distribution of reflection phase, the scattered wave front will have a planar equiphase surface with respect to the incident EM wave causing the reflected wave to be completely focussed in a particular direction. Therefore, if the pattern on the conducting plate is designed to have non-uniform distribution of reflection phase, the equiphase wave front will be disturbed and the impinging EM wave will be scattered randomly in different directions. This implies that the directional reflection in the specular direction will be minimized as the EM energy gets diffused in various directions. Further, the specular reflection can be completely eliminated by appropriate distribution of reflection phase of the conducting elements. ${ }^{54}$ This is the theoretical background behind the application of diffusion metasurfaces for RCSR (Fig. 15).

The scattered waves from different parts of the conducting surface will undergo destructive interference due to non-uniform phase shifts eventually leading to diffusion of the reflected waves. A comparatively huge phase swing is required to effectively diffuse the scattered energy into all possible directions with the magnitude of the normalized reflection coefficient of each meta-atom close to $0 \mathrm{~dB} .{ }^{61}$ Paquay et al. 62 demonstrated that a chessboard configuration (Fig. 16a) consisting of both perfect electric conductors (PEC) and artificial magnetic conductors (AMC) can reduce RCS due to destructive interference between the reflected waves from both units. PEC reflects the incident EM wave with a phase shift of $180^{\circ}$, whereas AMC reflects without any phase shift at the operating frequency. However, the bandwidth of this configuration is very narrow. Zhao et al. 63 achieved -10dB RCS reduction over slightly broader frequency range from $7.38 \mathrm{GHz}$ to $10.47 \mathrm{GHz}$ with an orthogonal arrangement of complementary split-ring resonator
(CSRR) based AMC blocks, as shown in Fig. 16b. Another chessboard-like configuration using Jerusalem cross-based AMC shown in Fig. 16c has been proposed in Ref. 64 with broadband RCS reduction from $14.5 \mathrm{GHz}$ to $21.8 \mathrm{GHz}$. In this case, the operational bandwidth has been found to be stable for incident angles to $10^{\circ}$. In a similar way, a chessboard model based on saltire arrow and E-shaped unit cells on $2.28 \mathrm{~mm}$ of RO4003 substrate (Fig. 16d) accomplished wideband RCSR over a frequency range of 9.4-23.28 GHz. ${ }^{65}$

An ultra-wideband polarization-insensitive diffusion metasurface has been presented in Ref. 66 where dimensionally different square ring meta-atoms (Fig. 17a) have been distributed appropriately using the principles of array synthesis and particle swarm optimization. The total processing time taken by the algorithm for optimization has been estimated as $83 \mathrm{~s}$. Further, Su et al. experimentally demonstrated that the ultra-thin metasurace fabricated using $\mathrm{RT} /$ duroid 5880 substrate (thickness $=1.57 \mathrm{~mm}$ ) have reflection coefficients less than 0.2 over the entire frequency range from $11.3 \mathrm{GHz}$ to $40 \mathrm{GHz}$ and over the range of incident angles from $0^{\circ}$ to $40^{\circ}$. Similar algorithm has been used in Ref. 67 for optimizing a single layer metasurface based on asymmetric double arrows (Fig. 17b), towards achieving monostatic as well as bistatic RCSR for co-polarized and cross-polarized incident waves. The optimized distribution achieved $10 \mathrm{~dB}$ monostatic RCSR from $7.5 \mathrm{GHz}$ to $22.5 \mathrm{GHz}$. Further, the performance was found to remain stable to an angle of incidence of $30^{\circ}$ for both TE as well as TM polarizations. On an identical note, Wang et al. 61 obtained RCSR greater than $10 \mathrm{~dB}$ (within the range of incident angles from $0^{\circ}$ to $45^{\circ}$ ) in the frequency range of $7-13$ GHz using a windmill shaped unit cell, ${ }^{68}$ shown in Fig. $17 \mathrm{c}$. Here, the far-field scattering algorithm took almost $10 \mathrm{~min}$ for each iteration in comparison with approximately $9 \mathrm{~h}$ for the same using numerical simulation. This methodology has been used for realizing low reflection surfaces in the $\mathrm{THz}$ domain ${ }^{69,70}$ as well. A representative flowchart summarizing the methodology for the optimization of diffusion
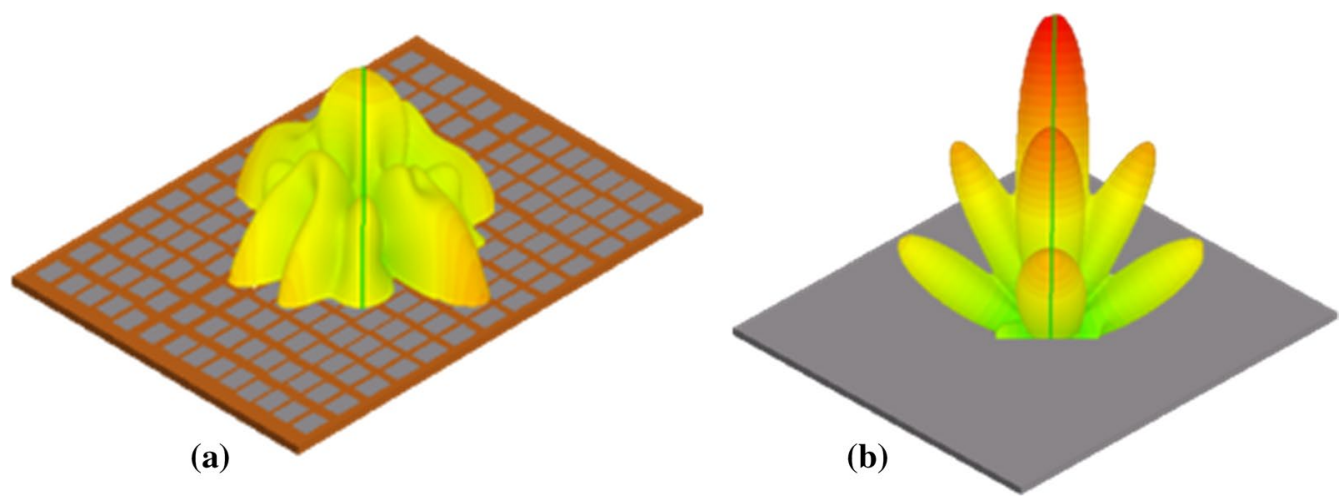

Fig. 15 Working principle of diffusion metasurfaces. (a) Metasurface with diffused reflections (b) Metal plate with specular reflection. 

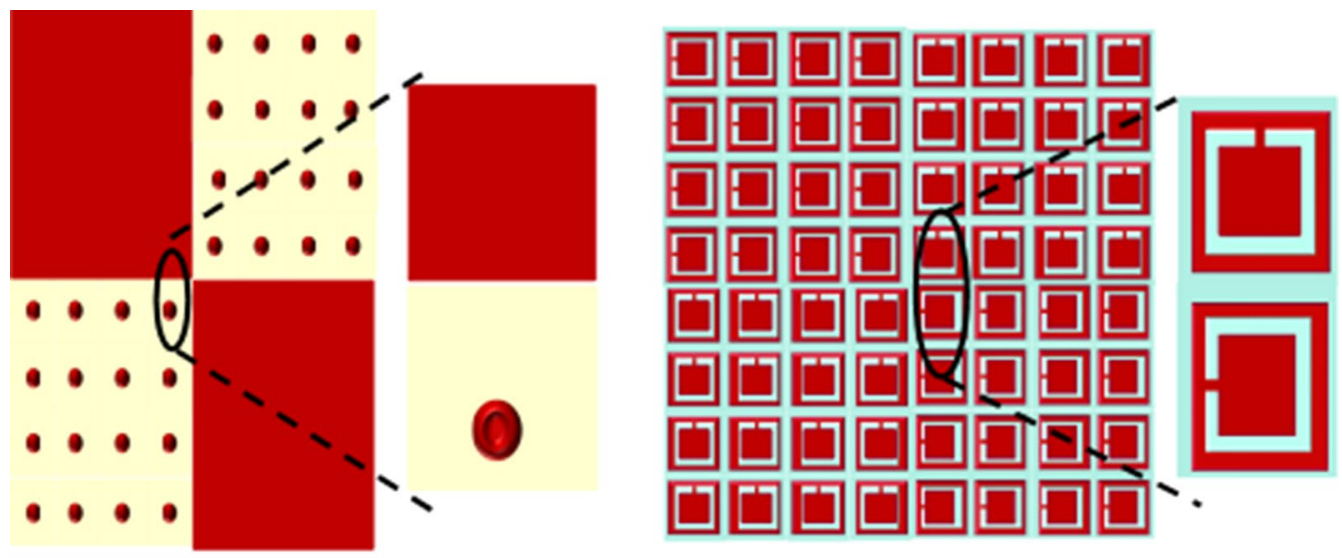

(b)

(a)
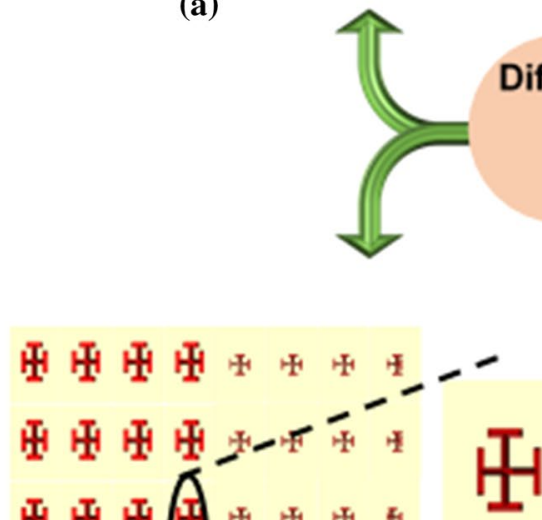

正正盟出出出

F

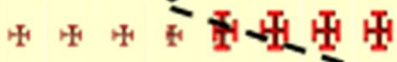

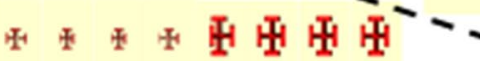

(c)
Diffusion metasurfaces with chess board configuration
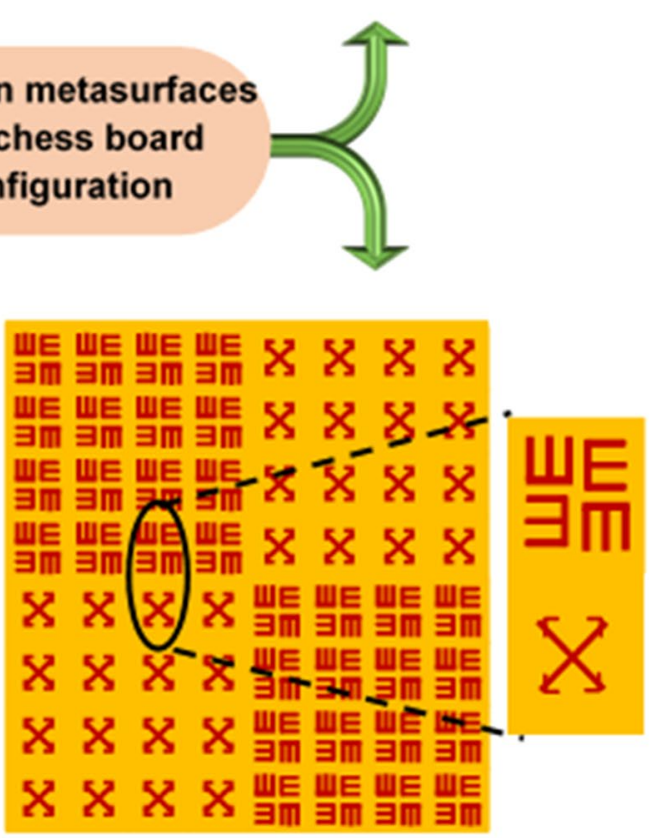

(d)

Fig. 16 Diffusion metasurfaces with chessboard configuration. (a) Data from 62 (b) Data from 63 (c) Data from 64 (d) Data from 65.

metasurfaces using PSO and array pattern synthesis (APS) is shown in Fig. 18.

Several reported works have combined diffused reflection mechanism along with other physical phenomena in order to achieve reduced RCS over broad frequency range. Diffused reflections in combination with surface wave conversion have been used in Ref. 71 for achieving more than $10 \mathrm{~dB}$ RCSR in the frequency range of 7.8-17.0 GHz. A 2D phase gradient metasurface (PGM) based on combined splitring resonator (CSRR), as shown in Fig. 19a has been used for accomplishing this. Further, polarization conversion and phase cancellation has been simultaneously accomplished in Ref. 12 using a chess board configuration based on concentric split ring meta atom as shown in Fig. 19b. This metasurface could achieve broad band RCS reduction (within the range of incident angles from $0^{\circ}$ to $30^{\circ}$ ) over a frequency range of $8.6-17.7 \mathrm{GHz}$.

Diffused reflections along with multiple resonances have been employed in Ref. 72 for realizing a polarisation insensitive metasurface with reduced RCS in the X-band over a range of incident angles from $0^{\circ}$ to $30^{\circ}$. The corresponding configuration is shown in Fig. 19c. Zhao et al. 73 proposed a 


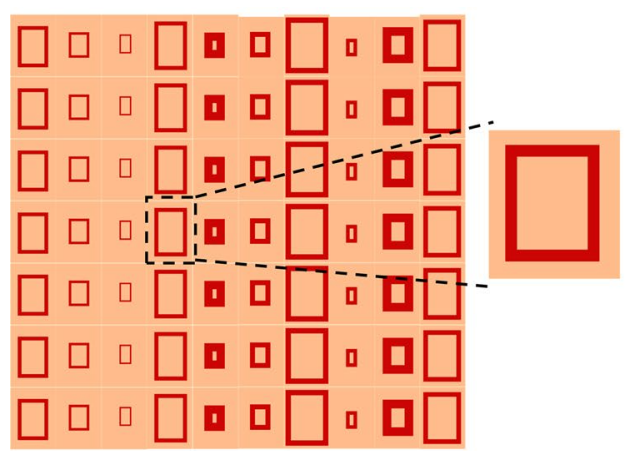

(a)

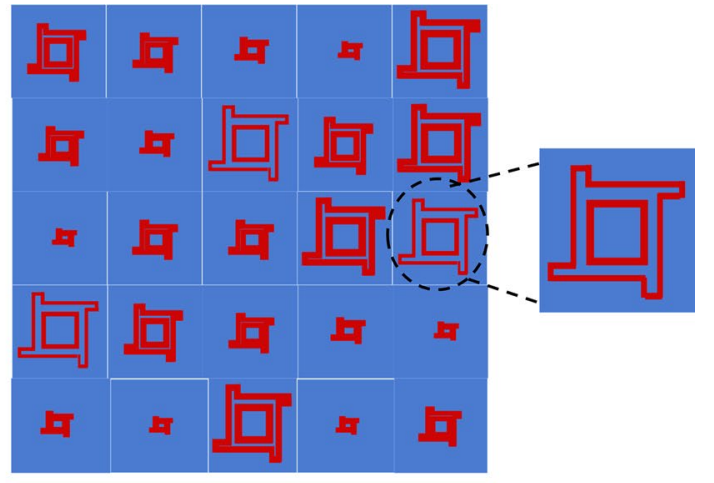

(c)

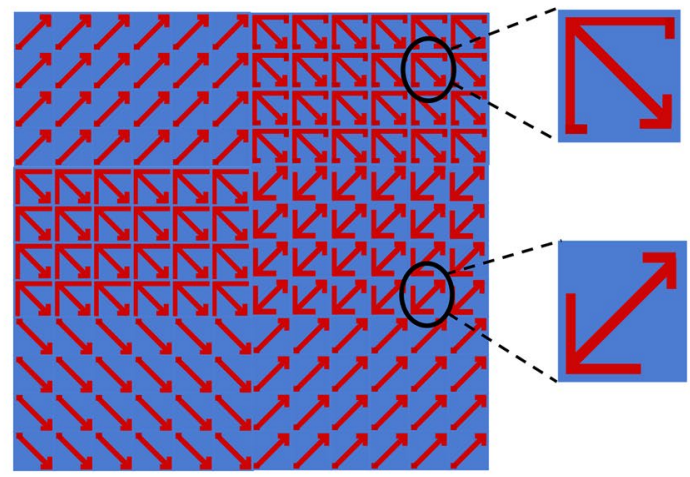

(b)

Fig. 17 Diffusion metasurfaces optimized using PSO and principles of array synthesis. (a) Data from 66 (b) Data from 67 (c) Data from 68.

flexible metasurface using an achiral rotationally asymmetric meta atom (Fig. 19d) for achieving RCSR in X-band. Here, metallic patches have been printed onto a polyimide film using flexible printed circuit (FPC) technology.

\section{Coding Metasurfaces for RCSR}

Coding/programmable metasurfaces present an efficient and powerful design approach for realizing unique functionalities by controlling the EM propagation. ${ }^{1,74}$ In this method, meta atoms with different reflection phases are arranged on the metasurface using coding sequences optimized for realizing a specific functionality. For instance, a 1-bit coding metasurface for RCSR will typically consist of two meta atoms assigned as ' 0 ' and ' 1 ' with their reflected wave fronts out of phase by $180^{\circ}$. The scattered waves from this digital metasurface can be tailored by optimizing the arrangement of ones and zeros on the surface using suitable optimization algorithm. Similarly, a 2-bit coding metasurface will have four digital elements. ${ }^{75}$ Yan et al. 76 proposed a flexible 2-bit coding metasurface composed of four elements ('00', '01', ' 10 ', and ' 11 ') based on double cross metallic line, as shown in Fig. 20a. The distribution of digital elements has been optimized using PSO and the resultant metasurface achieved reflection coefficients less than $-10 \mathrm{~dB}$ over a broad frequency range from $0.8 \mathrm{THz}$ to $1.5 \mathrm{THz}$. The metasurface also exhibited a stable frequency response for angles of incidence to $50^{\circ}$ for TM polarization. Liang et al. 77 achieved similar performance using a 1-bit polarization independent metasurface based on metallic ring resonator. Similarly, Zhang et al. 75 experimentally realized a 1-bit coding diffusion metasurface based on square ring patches as shown in Fig. 20b. The digital elements have been optimized to have a reflection phase difference of $180^{\circ} \pm 37^{\circ}$ and consequently achieved RCSR over a broad frequency band from $8.4 \mathrm{GHz}$ to $22.76 \mathrm{GHz}$. Array analysis and simulated annealing algorithm have been used in Ref. 78 to optimize the layout of another 1-bit coding metasurface based on a simple rectangular patch element, as shown in Fig. 20c. The optimized metasurface exhibited reduced RCS in the frequency ranges of 5.26-6.80 GHz and 5.56-6.82 GHz for $x$ - and $y$-polarizations, respectively. Similar configuration of meta atom has been used in Ref. 79 to realize a 1-bit coding metasurface (Fig. 20d) with reduced RCS in the $\mathrm{THz}$ regime. Here, the optimal layout has been generated based on Golay-Rudin-Shapiro (GRS) sequences. Further, a 3-bit coding metasurface based on a symmetric split ring unit cell (Fig. 20e) has been proposed ${ }^{80}$ with $10 \mathrm{~dB}$ co-polarized 
PSO Module

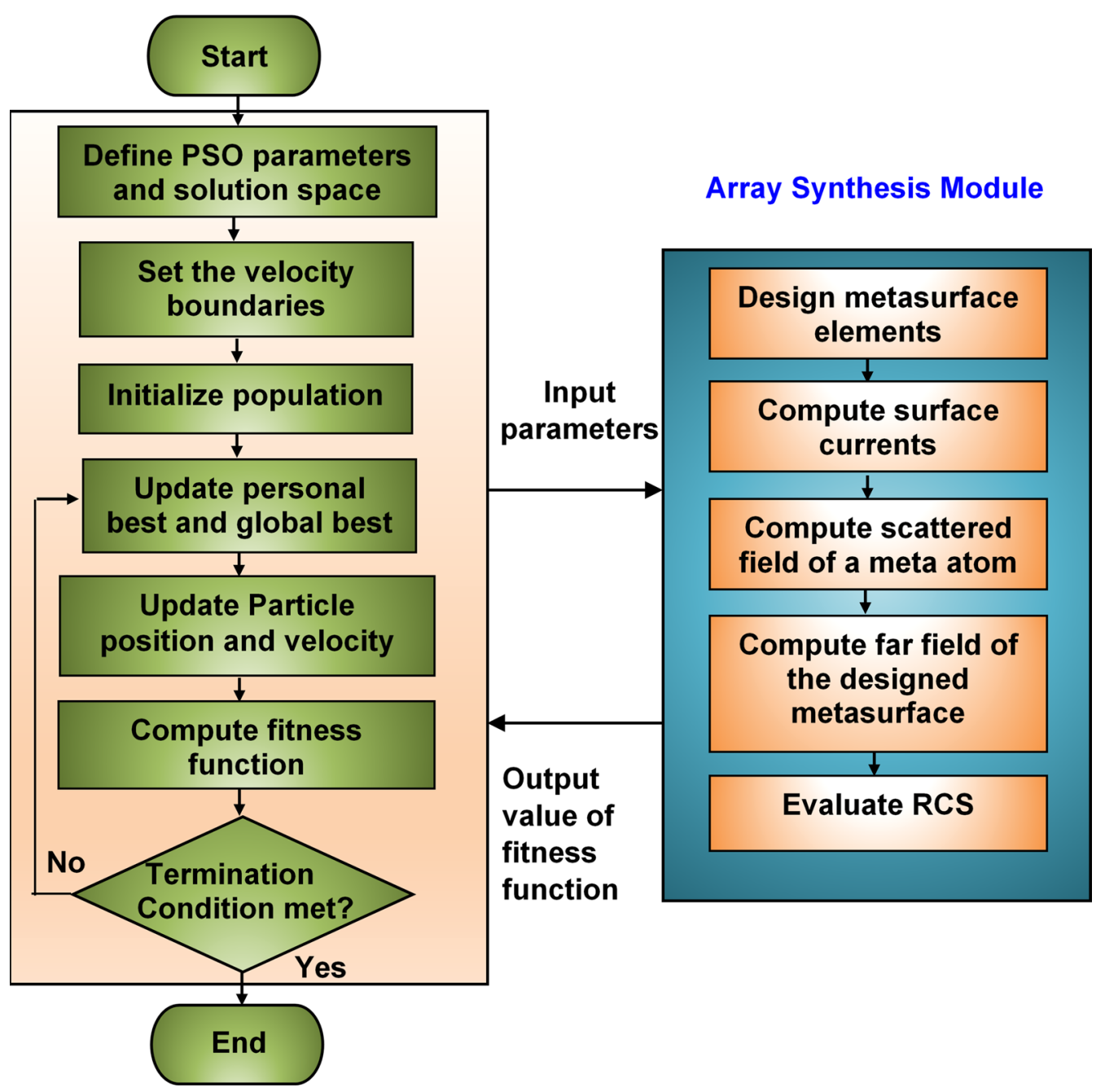

Fig. 18 Flowchart for the optimization of diffusion metasurfaces using PSO and array synthesis.

RCSR (within the range of incident angles from $0^{\circ}$ to $30^{\circ}$ ) from $8 \mathrm{GHz}$ to $18 \mathrm{GHz}$. Both 1-bit as well as 2-bit coding metasurfaces with varied functionalities under polarizations have been theoretically and experimentally demonstrated at $\mathrm{THz}$ frequencies. ${ }^{81}$ A summary of prospective metasurfaces discussed so far with significant RCS reduction over a broad band is presented in Table I. Further, Table II summarizes the prominent techniques used by researchers to obtain the optimal layout in the case of diffusion metasurfaces.

\section{Metasurfaces for RCS Reduction of Antennas}

Antennas form an integral part of the communication systems with multiple entities deployed on military vehicles for navigation, tracking, surveillance, etc. They contribute immensely to the total RCS of the vehicle owing to their functionality to efficiently transmit and receive signals. Therefore, reducing the scattering from antennas is extremely crucial in determining the overall stealth performance of the military assets. Metasurfaces have received significant research attention for this application due to their RCSR capabilities and ease of conformability. Su et al. 82 reduced the monostatic as well as bistatic RCS of a microstrip patch antenna array using a 1-bit coding metasurface (Fig. 21a). Significant monostatic RCSR has been observed from $6 \mathrm{GHz}$ to $7.6 \mathrm{GHz}$ and $9.5 \mathrm{GHz}$ to $26 \mathrm{GHz}$ while preserving its radiation characteristics and compactness. Likewise, $\mathrm{Li}$ et al. 83 reduced out-of-band scattering from a conventional microstrip patch antenna using a 2-bit coding metasurface as shown in Fig. 21b. The design is based on a chessboard configuration with two different AMCs used for obtaining a phase difference between $180^{\circ} \pm 37^{\circ}$ over broad band of frequencies. The optimized layout, generated using matrix type random coding, achieved more than $7 \mathrm{~dB}$ RCSR 


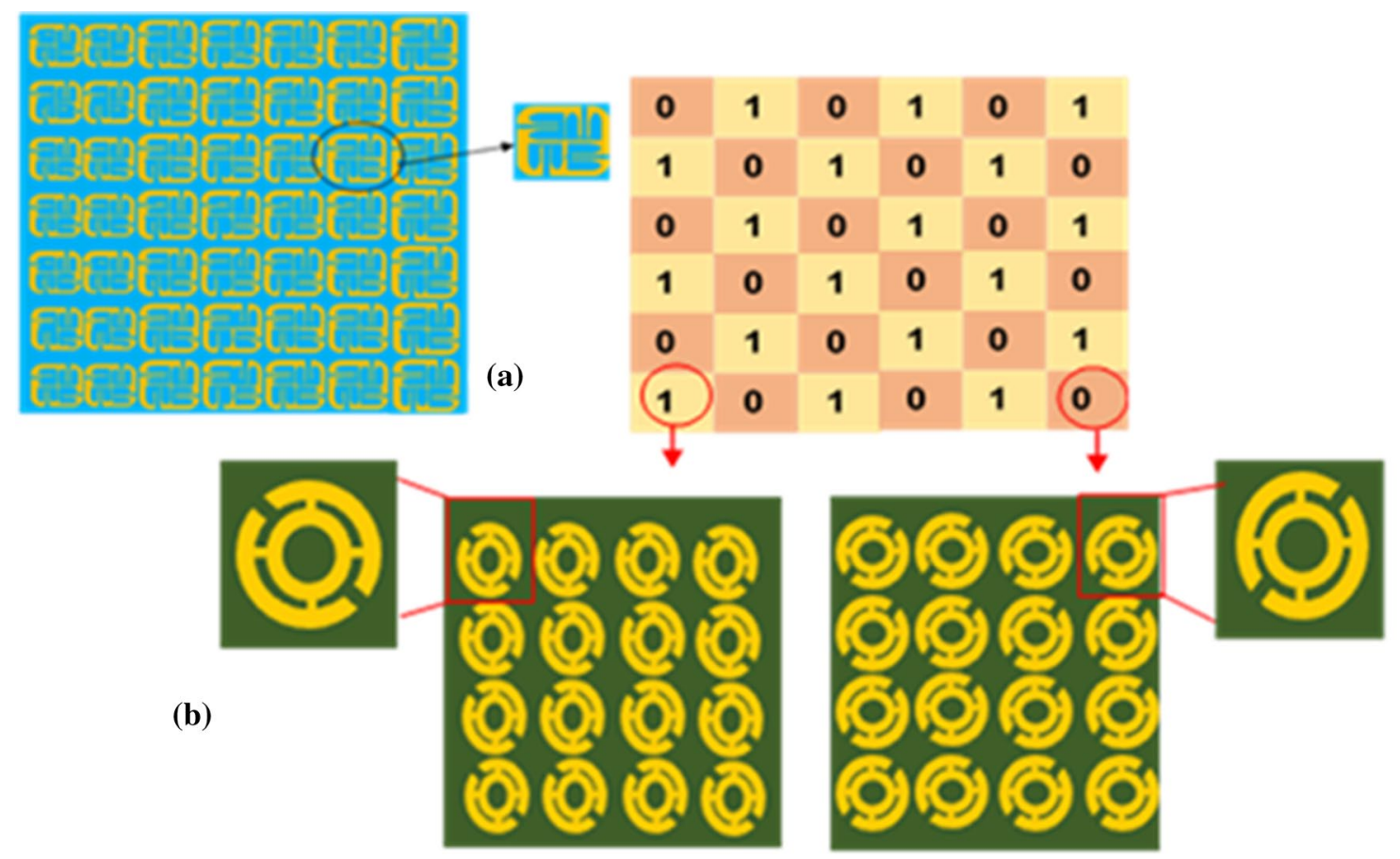

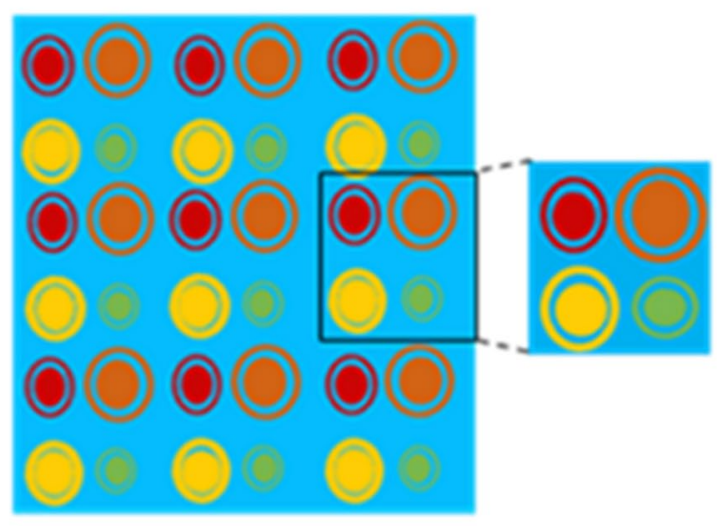

(c)

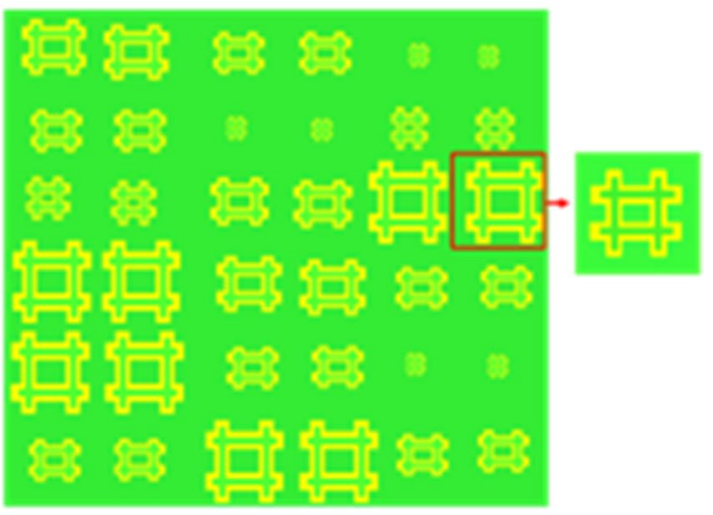

(d)

Fig. 19 Metasurfaces for RCSR based on diffusion of reflections and other physical phenomena. (a) Data from 71 (b) Data from 12 (c) Data from 72 (d) Data from 73.

from $12.2 \mathrm{GHz}$ to $19.8 \mathrm{GHz}$ without affecting the radiation performance at the operating frequency of $8.7 \mathrm{GHz}$. Further, RCS of a high gain Fabry-Perot antenna has been reduced in X-band using a 2-bit coding metasurface. ${ }^{84}$ Antenna elements based on polarization conversion metasurface (PCM) has been used ${ }^{85}$ to realize a circularly polarized (CP) antenna array with reduced RCS. Phase cancellation obtained due to the chessboard configuration of the antenna elements (Fig. 21c) aided in achieving both in-band as well as out-ofband RCSR from $5.7 \mathrm{GHz}$ to $7.7 \mathrm{GHz}$. In a similar way, a cross-polarization conversion metasurface ${ }^{86}$ has been used to reduce both in-band as well as out-of- band RCS of a microstrip patch antenna. It has also been observed that the gain of the proposed antenna improved by $2 \mathrm{~dB}$ with radiation characteristics remaining intact. Jia et al. 87 presented a metasurface based aperture coupled patch antenna (Fig. 21d) with 10-dB RCSR over a wide frequency band from $5.5 \mathrm{GHz}$ to $17.0 \mathrm{GHz}$. In addition, the gain of the proposed antenna got enhanced from $7.5 \mathrm{GHz}$ to $11.5 \mathrm{GHz}$ with almost $4.1 \mathrm{~dB}$ rise at $9.7 \mathrm{GHz}$.

A phase gradient metasurface (PGM) has been proposed in Ref. 88 to reduce the out-of-band RCS of a slot array antenna operating in the frequency range of 4.12-4.26 GHz. The PGM, loaded on the antenna as a superstrate, obtained significant RCSR from $7 \mathrm{GHz}$ to $16.8 \mathrm{GHz}$. The reduced scattering can be attributed to the anomalous as well as diffused reflection characteristics of the PGM. Further, an absorber loaded microstrip antenna with a tunable PGM 


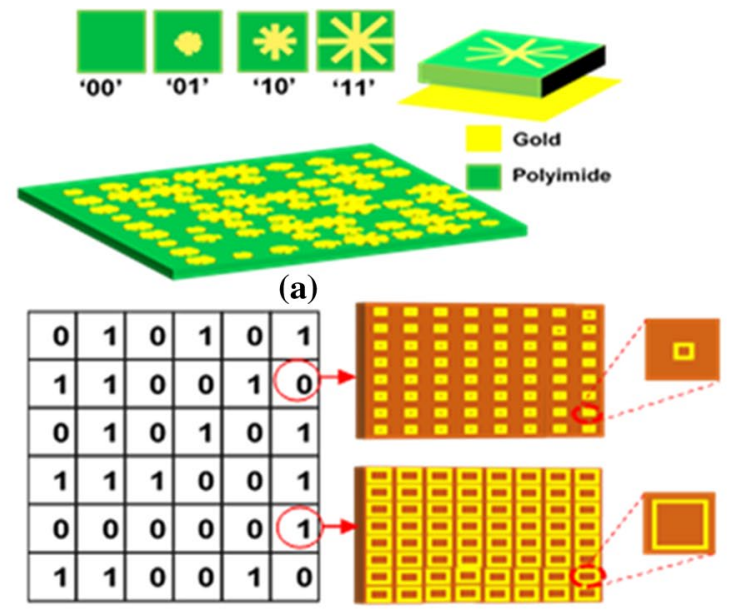

(b)

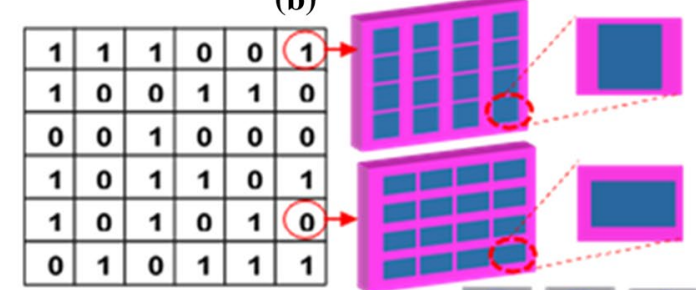

(c)

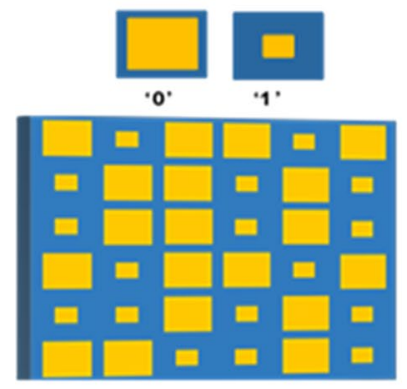

(d)

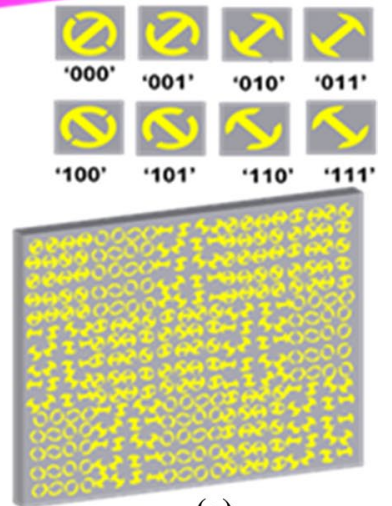

(e)

Fig. 20 Coding metasurfaces used for RCS reduction. (a) Data from 76 (b) Data from 75 (c) Data from 78 (d) Data from 79 (e) Data from 80.

Table I Summary of prospective metasurfaces with broadband $\mathrm{RCS}$ reduction based superstrate proposed by $\mathrm{Yu}$ et al. 89 achieved beam steering, RCSR and gain improvement in the operating band of 4.2-4.55 GHz. A resistive metasurface based superstrate which is transparent in the operational band of a patch antenna array and absorptive for out-of-band as well as cross-polarized incidence has been proposed by Chen et al. 90. RCSR for co-polarized waves has been observed from $5 \mathrm{GHz}$ to $9.1 \mathrm{GHz}$ in the lower band and $11.4-16.1 \mathrm{GHz}$ in the upper band. On the other hand, the achieved cross-polarized RCS reduction bandwidth is from $5.5 \mathrm{GHz}$ to $16.3 \mathrm{GHz}$. Liu et al. 91 experimentally realized a patch antenna with high gain and reduced RCS based on a holographic metasurface (HM), as shown in Fig. 21e. The antenna gain is obtained as $10 \mathrm{~dB}$ owing to the parasitic nature of HM. A wideband RCSR from $5.5 \mathrm{GHz}$ to $21.5 \mathrm{GHz}$ has been achieved due to its ability to transform the propagating wave into a surface wave. A low RCS microstrip antenna for conformal applications has been proposed in Ref. 92 where fan shaped -atoms have been used to accomplish phase cancellation. The proposed antenna obtained broadband RCSR from $5 \mathrm{GHz}$ to $9.5 \mathrm{GHz}$. A summary of low RCS antennas based on metasurfaces is presented in Table III.

\section{Recent Trends and Perspectives}

The conventional workflow for the design of metasurfaces predominantly involves the brute-force approach starting with preliminary EM modelling followed by extensive parameter optimization using evolutionary algorithms such as PSO and GA. The main drawback of these algorithms is that numerous EM simulations have to be carried out before finalizing the best possible design. This is computationally exhaustive as well as time consuming. The situation becomes worse in the case of designs with complex metaatoms where the number of parameters to be optimized are exceedingly high. However, researchers accept this complex and resource consuming design process as it is often the only

\begin{tabular}{lllc}
\hline Reference & Configuration of unit cell & $\begin{array}{l}\text { Total thickness } \\
(\mathrm{mm})\end{array}$ & $\begin{array}{l}\text { Frequency range with } \\
\text { 10 dB RCSR (GHz) }\end{array}$ \\
\hline Ghosh et al. 50 & Split ring & 2 & $7.8-12.2$ \\
Zhao et al. 63 & CSRR & 2 & $7.3-10.4$ \\
Su et al. 80 & Spit ring and cut wire & 3 & $7.9-21$ \\
Wang et al. 61 & Wind mill & 4 & $7-13$ \\
Galarregui et al.64 & Jerusalem cross & 1.27 & $14.5-21.8$ \\
Esmaeli et al. 65 & Saltire arrow and E-patch & 2.28 & $9.4-23.2$ \\
Lu et al. 67 & Arrow and square cut patch & 3 & $7.5-22.5$ \\
Li et al. 71 & Split ring & 2.5 & $7.8-17$ \\
Fang et al. 12 & Split ring & 3 & $8.6-17.7$ \\
Song et al. 72 & Disc with concentric ring & 3 & $7-12$ \\
Zhao et al. 73 & '\#' shaped ring with square ring & 4 & $7.6-12.1$ \\
\hline
\end{tabular}


Table II Summary of techniques used to obtain the optimal layout in the case of diffusion metasurfaces

\begin{tabular}{lllcl}
\hline Reference & Type of unit cell & Optimization technique & $\begin{array}{c}\text { Operational freq. } \\
\text { range (GHz) }\end{array}$ & $\begin{array}{l}\text { 10 dB RCSR } \\
\text { bandwidth } \\
(\mathrm{GHz})\end{array}$ \\
\hline Su et al. 66 & Square ring & PSO and APS & $11.3-51.3$ & 40 \\
Lu et al. 67 & Double arrow & PSO and APS & $7.5-22.5$ & 15 \\
Zhang et al. 69 & Square patch & PSO and APS & $900-1600$ & 700 \\
Dong et al. 70 & '\#’ and square rings & PSO and APS & $1000-1800$ & 800 \\
Wang et al. 61 & Windmill & PSO and APS & $7-14$ & 7 \\
Yan et al. 76 & Double cross line & PSO & $800-1500$ & 700 \\
Zhao et al. 78 & Rectangular patch & Annealing algorithm & $4-8$ & 4 \\
Moccia et al. 79 & Square patch & GRS sequence & $800-1600$ & 800 \\
\hline
\end{tabular}

feasible solution to obtain a high-quality design. With rapid developments in data science, cutting-edge data processing technologies and affordable data storage, machine learning algorithms can be effectively used to minimize the role of computationally expensive EM simulators in the design of metasurfaces for stealth applications.

A deep learning neural network has been proposed in Ref. 93 for the design of anisotropic coding metasurfaces. Artificial neural networks (ANNs), popularly known to have functionality similar to that of a human brain, are data processing frameworks that can predict the solution to a problem by learning from a set of similar problems. The accuracy of the predicted solution largely depends upon the quality of training, i.e., the amount of data and type of algorithm. The proposed deep learning methodology in Ref. 93 could extract the relation between unit cell pattern and reflection characteristics using a training data set containing 70,000 examples in place of the $2^{64}$ combinations practically possible for a $16 \times 16$ lattice. The model has been then used for predicting the metasurface pattern for accomplishing multibeam properties.

Deep learning approach has also been used for the modelling of all-dielectric metasurfaces in Ref. 94. Shan et al. 95 proposed a convolutional neural network for the design of coding metasurfaces for beam forming applications. Recently, a fully automatic metasurface design framework based on deep learning approach has been proposed in Ref. 96. The proposed framework named 'Reactive' is capable of predicting the metasurface pattern for achieving a particular design target. For the purpose of illustration, a triple-band absorber has been designed and the time taken has been reduced by almost $98 \%$ with respect to conventional design approach. Xiao et al. 97 proposed a similar model in the terahertz band where sufficient accuracy has been achieved with less number of training examples. Further, an integrated hardware platform has been designed in Ref. 98 where a network of electronic controllers generates the appropriate metasurface on the basis of external commands requesting for a specific EM functionality.

\section{Conclusion}

Metasurfaces are gradually replacing their 3D counterparts, i.e., metamaterials on account of their attractive features like reduced weight penalties, less space consumption, low losses and ease of fabrication. These features in combination with their capability to manipulate EM waves make them excellent choices for accomplishing stealth features on various military platforms. In this regard, the present paper has given a concise overview on the recent advancements in the field of metasurfaces for low observable applications. The theoretical background has been explained with supporting formulation. Further, the different kinds of metasurfaces that have been reported in open domain for RCSR including diffusion metasurfaces, coding metasurfaces and low RCS antennas based on metasurfaces have been elaborately reviewed. It is apparent from the reported works that metasurfaces can reduce scattering over a broad range of frequencies for a wide range of incident angles and arbitrary polarizations with reduced thickness. The various techniques used for the design and optimization of metasurfaces have also been mentioned including the current trend of using deep learning methodology for the same. The metasurfaces presented here are by no means the only ones capable of accomplishing RCSR. However, they give an indication on how metasurfaces can be designed for specific constraints (frequency range and thickness) and on the techniques that can provide optimized performance. 


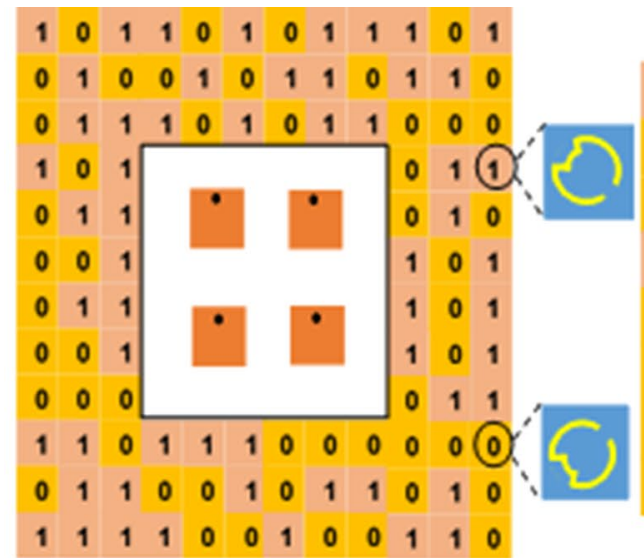

(a)

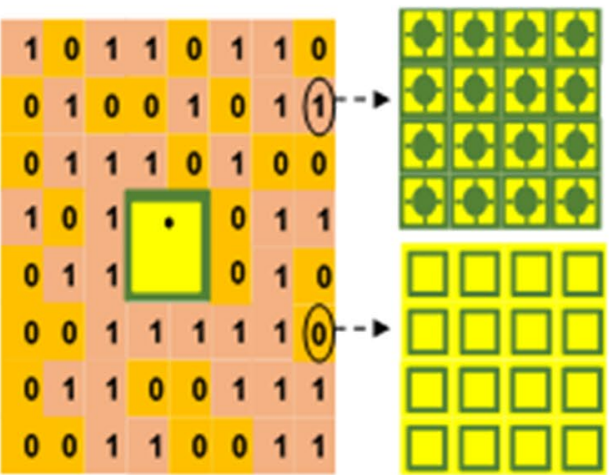

(b)

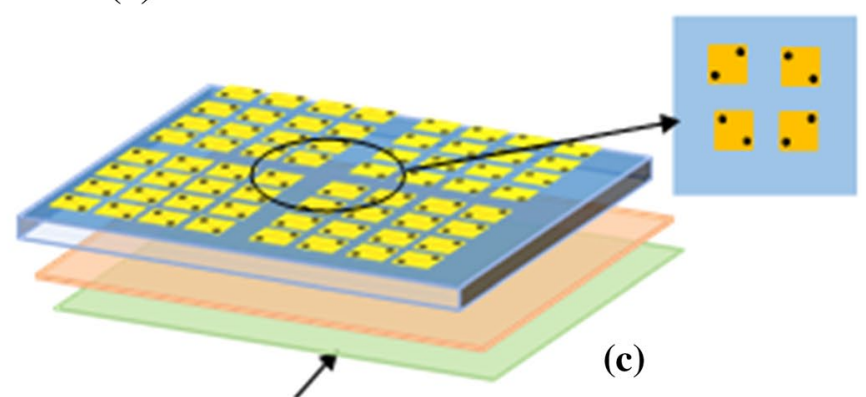

Feeding network

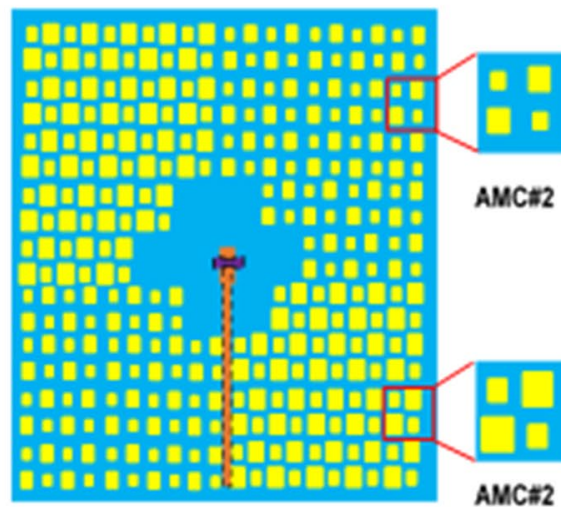

(d)

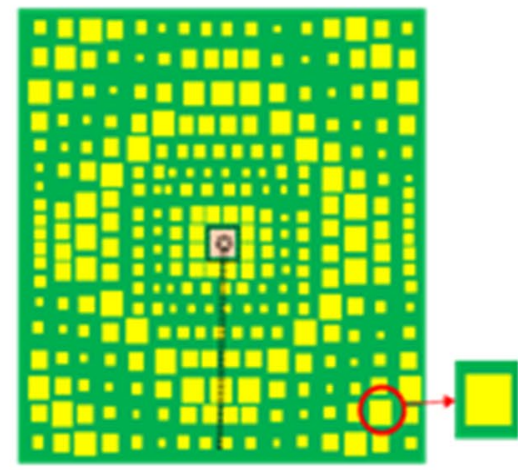

(e)

\section{F4B $\square$ RO $3003 \square$ FR4 Arlon AD 450 F2B $^{\circ}$. . Metal pin PEC}

Fig. 21 Low RCS antennas based on metasurfaces. (a) Data from 82 (b) Data from 83 (c) Data from 85 (d) Data from 87 (e) Data from 91.

Table III Summary of low RCS antennas based on metasurfaces

\begin{tabular}{lll}
\hline Reference & $\begin{array}{l}\text { In band RCSR band- } \\
\text { width }(\mathrm{GHz})\end{array}$ & $\begin{array}{l}\text { Out of band RCSR } \\
\text { bandwidth }(\mathrm{GHz})\end{array}$ \\
\hline Su et al. 82 & $9.5-26$ & $6.72-7.6$ \\
Li et al. 83 & - & $12.2-19.8$ \\
Zhang et al. 85 & $5.7-7.7$ & - \\
Jia et al. 87 & - & $5.5-17$ \\
Liu et al. 91 & $9.7-10.7$ & $5.5-21.5$ \\
\hline
\end{tabular}

Acknowledgments This work was supported by the Directorate of Extramural Research and Intellectual Property Rights, Defence Research and Development Organization (DRDO), India.

Conflict of interest The authors declare that they have no conflict of interest. 


\section{References}

1. T.J. Cui, S. Liu, and L. Zhang, J. Mater. Chem. C 5, 3644 (2017).

2. L. Liu, X. Zhang, M. Kenney, X. Su, N. Xu, C. Ouyang, Y. Shi, J. Han, W. Zhang, and S. Zhang, Adv. Mater. 26, 5031 (2014).

3. C. Du, D. Zhou, H.-H. Guo, Y.-Q. Pang, H.-Y. Shi, W.-F. Liu, J.-Z. Su, C. Singh, S. Trukhanov, A. Trukhanov, L. Panina, and Z. Xu, Nanoscale 12, 9769 (2020).

4. K. Achouri, B.A. Khan, S. Gupta, G. Lavigne, M.A. Salem, and C. Caloz, EPJ Appl. Metamater. 2, 12 (2015).

5. S.S. Grabchikov, A.V. Trukhanov, S.V. Trukhanov, I.S. Kazakevich, A.A. Solobay, V.T. Erofeenko, N.A. Vasilenkov, O.S. Volkova, and A. Shakin, J. Magn. Mater. 398, 49 (2016).

6. A.V. Trukhanov, V.G. Kostishyn, L.V. Panina, S.H. Jabarov, V.V. Korovushkin, S.V. Trukhanov, and E.L. Trukhanova, Ceram. Int. 43, 12822 (2017).

7. S.V. Trukhanov, A.V. Trukhanov, V.G. Kostishin, L.V. Panina, I.S. Kazakevich, V.A. Turchenko, V.V. Oleinik, E.S. Yakovenko, and L.Y. Matsui, JETP 123, 461 (2016).

8. S.V. Trukhanov, A.V. Trukhanov, V.G. Kostishyn, L.V. Panina, A.V. Trukhanov, V.A. Turchenko, D.I. Tishkevich, E.L. Trukhanova, O.S. Yakovenko, L.Y. Matzui, D.A. Vinnik, and D.V. Karpinsky, J. Phys. Chem. Solids 111, 142 (2017).

9. P.-J. Wang, D. Zhou, J. Li, L.-X. Pangc, W.-F. Liu, J.-Z. Su, C. Singh, S. Trukhanov, and A. Trukhanov, Nano Energy 78, 105247 (2020).

10. S. Bukhari, J.Y. Vardaxoglou, and W. Whittow, Appl. Sci. 9, 2727 (2019).

11. K. Achouri and C. Caloz, Nanophotonics 7, 1095 (2018).

12. W. Fang, X. Xie, S. Sun, Y. Wang, D. Fan, X. Liu, and P. Chen, Int. J. RF Microw. Comput. Aided Eng. (2019). https://doi.org/10. 1002/mmce.22069.

13. D. Ramaccia, D.L. Sounas, A. Alù, A. Toscano, and F. Bilotti, IEEE Trans. Antennas Propag. 68, 1607 (2020).

14. D. Ramaccia, F. Bilotti, A. Toscano, D.L. Sounas, and A. Alù, in IEEE International Symposium on Antennas and Propagation and USNC/URSI National Radio Science Meeting (2017), p. 73.

15. Z. Seyedrezaei, B. Rejaei, and M. Memarian, Opt. Express 28, 6378 (2020).

16. K. Lee, J. Son, B. Kang, J. Park, F. Rotermund, and B. Min, in $42 n d$ International Conference on Infrared, Millimeter, and Terahertz Waves (2017), p. 1.

17. F. Bi, Z. Ba, and X. Wang, Opt. Express 26, 25693 (2018).

18. X. Luo, Sci. China Phys. Mech. Astron. 58, 594201 (2015). https:// doi.org/10.1007/s11433-015-5688-1.

19. H.T. Chen, A.J. Taylor, and N. Yu, Rep. Prog. Phys. 79, 076401 (2016).

20. N. Yu, P. Genevet, M.A. Kats, F. Aieta, J.P. Tetienne, F. Capasso, and Z. Gaburro, Science 334, 333 (2011).

21. C.L. Holloway, E.F. Kuester, J.A. Gordon, J. Ohara, J. Booth, and D.R. Smith, IEEE Antennas Propag. Mag. 54, 10 (2012).

22. X. Luo, M. Pu, X. Ma, and X. Li, Int. J. Antennas Propag. (2015). https://doi.org/10.1155/2015/204127.

23. H. Hsiao, C.H. Chu, and D.P. Tsai, Small Methods (2017). https:// doi.org/10.1002/smtd.201600064.

24. N. Yu, F. Aieta, P. Genevet, M.A. Kats, Z. Gaburro, and F. Capasso, Nano Lett. 12, 6328 (2012).

25. F. Ding, Y. Yang, R.A. Deshpande, and S.I. Bozhevolnyi, Nanophotonics 7, 1129 (2018).

26. A. Pors, O. Albrektsen, I.P. Radko, and S.I. Bozhevolnyi, Sci. Rep. 3, 2155 (2013).

27. C. Liu, Y. Bai, Q. Zhao, Y. Yang, H. Chen, J. Zhou, and L. Qiao, Sci. Rep. 6, 34819 (2016).

28. C. Pfeiffer and A. Grbic, Phys. Rev. Lett. 110, 197401 (2013).
29. M. Londono, A. Sayanskiy, J.L. Araque-Quijano, S.B. Glybovski, and J.D. Baena, Phys. Rev. Appl. 10, 034026 (2018).

30. G. Xu, S.V. Hum, and G.V. Eleftheriades, IEEE Trans. Antennas Propag. 67, 6935 (2019).

31. A. Leitis, A. Hebler, S. Wahl, M. Wuttig, T. Taubner, A. Tittl, and H. Altug, Adv. Funct. Mater. (2020). https://doi.org/10.1002/adfm. 201910259.

32. R. Alaee, M. Albooyeh, A. Rahimzadegan, M.S. Mirmoosa, Y.S. Kivshar, and C. Rockstuhl, Phys. Rev. B (2015). https://doi.org/ 10.1103/PhysRevB.92.245130.

33. M. Odit, P. Kapitanova, P. Belov, R. Alaee, C. Rockstuhl, and Y.S. Kivshar, Appl. Phys. Lett. 108, 1 (2016). https://doi.org/ 10.1063/1.4953023.

34. P. Kapitanova, M. Odit, M. Danaeifar, A. Sayanskiy, P. Belov, A. Miroshnichenko, and Y. Kivshar, in Proceedings of the 47th European Microwave Conference (2017), p. 476.

35. Z. Wang, S. He, Q. Liu, and W. Wang, Opt. Commun. 367, 144 (2016).

36. S.M. Kamali, A. Arbabi, E. Arbabi, Y. Horie, and A. Faraon, Nat. Commun. (2016). https://doi.org/10.1038/ncomms11618.

37. F. Ding, P. Genevet, and S. Bozhevolnyi, Appl. Sci. (2018). https://doi.org/10.3390/app8101727.

38. M. Chen, L. Jiang, and W. Sha, Appl. Sci. (2018). https://doi. org/10.3390/app8030362.

39. B. Liu, K. Song, and J. Xiao, Adv. Condens. Matter Phys. (2019). https://doi.org/10.1155/2019/2329168.

40. S.D. Campbell, D. Sell, R.P. Jenkins, E.B. Whiting, J.A. Fan, and D.H. Werner, Opt. Mater. Express 9, 1842 (2019).

41. R.L. Haupt and D.H. Werner, Genetic Algorithms in Electromagnetics (New York: Wiley, 2007).

42. F. Bociort, A. Serebriakov, and J. Braat, in International Optical Design Conference OSA Technical Digest Series (2002), p. ITuA5.

43. Y. Dang, S. Yin, F. Deng, J. Qi, and H. Li, in International Symposium of Antennas and Propagation (2018), p. 67.

44. J. Robinson and Y. Rahmat-Samii, IEEE Trans. Antennas Propag. 52, 397 (2004).

45. A.V. Kildishev, U.K. Chettiar, Z. Liu, V.M. Shalaev, D.-H. Kwon, Z. Bayraktar, and D.H. Werner, J. Opt. Soc. Am. B 24, A34 (2007).

46. P.E. Sieber and D.H. Werner, Opt. Express 22, 32371 (2014).

47. S. Ghosh, S. Bhattacharyya, Y. Kaiprath, and K.V. Srivastava, J. Appl. Phys. 115, 104503 (2014).

48. H. Hao, S. Du, and T. Zhang, PIER M 81, 97 (2019).

49. Y. Jia, Y. Liu, Y.J. Guo, K. Li, and S.X. Gong, IEEE Trans. Antennas Propag. 64, 179 (2016).

50. S. Ghosh, S. Bhattacharyya, D. Chaurasiya, and K.V. Srivastava, IEEE Antennas Wirel. Propag. Lett. 14, 1172 (2015).

51. S. Li, J. Gao, X. Cao, Z. Zhang, Y. Zheng, and C. Zhang, Opt. Express (2015). https://doi.org/10.1364/OE.23.003523.

52. S. Bhattacharyya, S. Ghosh, K.V. Srivastava, and D. Chaurasiya, IET Microw. Antennas Propag. 9, 1160 (2015).

53. A. Sharma, R. Panwar, and R. Khanna, IEEE Magn. Lett. 10, 2101905 (2019).

54. X.M. Yang, G.L. Jiang, X.G. Liu, and C.X. Weng, Int. J. Antennas Propag. (2015). https://doi.org/10.1155/2015/560403.

55. Y.C. Hou, W.J. Liao, C.C. Tsai, and S.H. Chen, IEEE Trans. Antennas Propag. 64, 1859 (2016).

56. Y. Pang, H. Cheng, Y. Zhou, and J. Wang, J. Phys. D Appl. Phys. 45, 215104 (2012).

57. A. Arbabi, Y. Horie, M. Bagheri, and A. Faraon, Nat. Nanotechnol. (2015). https://doi.org/10.1038/NNANO.2015.186.

58. M.I. Khan, Z. Khalid, and F.A. Tahir, Sci. Rep. 9, 4552 (2019).

59. H. Chen, J. Wang, H. Ma, S. Qu, Z. Xu, A. Zhang, M. Yan, and Y. Li, J. Appl. Phys. 115, 154504 (2014).

60. Y. Jia, Y. Liu, Y.J. Guo, K. Li, and S. Gong, IEEE Trans. Antennas Propag. 65, 3291 (2017). 
61. K. Wang, J. Zhao, Q. Cheng, D.S. Dong, and T.J. Cui, Sci. Rep. 4, 5935 (2014).

62. M. Paquay, J.C. Iriarte, I. Ederra, R. Gonzalo, and P. de Maagt, IEEE Trans. Antennas Propag. 55, 3630 (2007).

63. Y. Zhao, X.Y. Cao, J. Gao, and W.Q. Li, Microw. Opt. Technol. Lett. 56, 158 (2014).

64. J.C.I. Galarregui, A.T. Pereda, J.L.M.D. Falcon, I. Ederra, R. Gonzalo, and P.D. Maagt, IEEE Trans. Antennas Propag. 61, 6136 (2013).

65. S. Esmaeli and S. Sedighy, Electron. Lett. 52, 70 (2016).

66. J. Su, Y. Lu, H. Zhang, Z. Li, Y. Yang, Y. Che, and K. Qi, Sci. Rep. 7, 42286 (2017).

67. Y. Lu, J. Su, J. Liu, Q. Guo, H. Yin, Z. Li, and J. Song, IEEE Trans. Antennas Propag. 67, 4936 (2019).

68. J. Chen, Q. Cheng, J. Zhao, D.S. Dong, and T.J. Cui, PIER 146, 71 (2014).

69. Y. Zhang, L. Liang, J. Yang, Y. Feng, B. Zhu, J. Zhao, T. Jiang, B. Jin, and W. Liu, Sci. Rep. 6, 26875 (2016).

70. D.S. Dong, J. Yang, Q. Cheng, J. Zhao, L.H. Gao, S.J. Ma, S. Liu, H.B. Chen, Q. He, W.W. Liu, Z. Fang, L. Zhou, and T.J. Cui, Adv. Opt. Mater. 3, 1405 (2015).

71. Y. Li, J. Zhang, S. Qu, J. Wang, H. Chen, Z. Xu, and A. Zhang, Appl. Phys. Lett. 104, 221110 (2014).

72. Y.C. Song, J. Ding, C.J. Guo, Y.H. Ren, and J.K. Zhang, IEEE Antennas Wirel. Propag. Lett. 15, 329 (2016).

73. J. Zhao, B. Sima, N. Jia, C. Wang, B. Zhu, T. Jiang, and Y. Feng, Opt. Express 24, 27849 (2016).

74. T.J. Cui, M.Q. Qi, X. Wan, J. Zhao, and Q. Cheng, Light Sci. Appl. (2014). https://doi.org/10.1038/lsa.2014.99.

75. H. Zhang, Y. Lu, J. Su, Z. Li, J. Liu, and Y.L. Yang, Electron. Lett. 53, 187 (2017).

76. X. Yan, L. Liang, J. Yang, W. Liu, X. Ding, D. Xu, Y. Zhang, T. Cui, and J. Yao, Opt. Express 23, 29128 (2015).

77. L. Liang, M. Qi, J. Yang, X. Shen, J. Zhai, W. Xu, B. Jin, W. Liu, Y. Feng, C. Zhang, H. Lu, H.T. Chen, L. Kang, W. Xu, J. Chen, T.J. Cui, P. Wu, and S. Liu, Adv. Opt. Mater. 3, 1374 (2015).

78. Y. Zhao, X. Cao, J. Gao, Y. Sun, H. Yang, X. Liu, Y. Zhou, T. Han, and W. Chen, Sci. Rep. 6, 23896 (2016).

79. M. Moccia, C. Koral, G.P. Papari, S. Liu, L. Zhang, R.Y. Wu, G. Castaldi, T.J. Cui, V. Galdi, and A. Andreone, Sci. Rep. 8, 11908 (2018).

80. P. Su, Y. Zhao, S. Jia, W. Shi, and H. Wang, Sci. Rep. 6, 20387 (2016).

81. S. Liu, T.J. Cui, Q. Xu, D. Bao, L. Du, X. Wan, W.X. Tang, C. Ouyang, X.Y. Zhou, H. Yuan, H.F. Ma, W.X. Jiang, J. Han, W. Zhang, and Q. Cheng, Light Sci. Appl. (2016). https://doi.org/10. 1038/lsa.2016.76.
82. J. Su, C. Kong, Z. Li, H. Yin, and Y. Yang, Electron. Lett. 53, 1088 (2017).

83. J. Li, T.A. Khan, X. Meng, J. Chen, G. Peng, and A. Zhang, IET Microw. Antennas Propag. 13, 1719 (2019).

84. L. Zhang, X. Wan, S. Liu, J.Y. Yin, Q. Zhang, H.T. Wu, and T.J. Cui, IEEE Trans. Antennas Propag. 65, 3374 (2017).

85. W. Zhang, Y. Liu, and Y. Jia, IEEE Antennas Wireless Propag. Lett. 18, 1453 (2019).

86. M.K.T. Al-Nuaimi and Y. He, in IEEE International Symposium on Antennas and Propagation and USNC-URSI Radio Science Meeting (2019), pp. 1907-1908.

87. X.Q. Jia, Y.J. Zheng, X.Y. Cao, J. Gao, Q. Chen, and Y.Q. Fu, AIP Adv. 9, 075103 (2019).

88. W. Zhang, Y. Liu, S. Gong, J. Wang, and Y. Jiang, IEEE Antennas Wirel. Propag. Lett. 17, 2193 (2018).

89. J. Yu, W. Jiang, and S. Gong, IEEE Antennas Wirel. Propag. Lett. 18, 2016 (2019).

90. Q. Chen, M. Guo, D. Sang, Z. Sun, and Y. Fu, IEEE Antennas Wirel. Propag. Lett. 18, 1223 (2019).

91. Y. Liu, N. Li, Y. Jia, W. Zhang, and Z. Zhou, IEEE Antennas Wirel. Propag. Lett. 18, 492 (2019).

92. T. Li, H. Yang, Y. Lei, S. Li, X. Cao, and D. Sun, in International Applied Computational Electromagnetics Society Symposium (2019).

93. Q. Zhang, C. Liu, X. Wan, L. Zhang, S. Liu, Y. Yang, and T.J. Cui, Adv. Theory Simul. (2018). https://doi.org/10.1002/adts.20180 0132.

94. S. An, C. Fowler, M. Y. Shalaginov, Y. Zhang, P. Su, M. Kang, B. Zheng, H. Tang, H. Li, A. M. Agarwal, C. R. Blaeine, K. A. Richadson, T. Gu, J. Hu, and H. Zhang, in International Applied Computational Electromagnetics Society Symposium (ACES) (2019).

95. T. Shan and M. Li, in IEEE International Symposium on Antennas and Propagation and USNC-URSI Radio Science Meeting (2019).

96. T. Qiu, X. Shi, J. Wang, Y. Li, S. Qu, Q. Cheng, T. Cui, and S. Sui, Adv. Sci. (2019). https://doi.org/10.1002/advs.201900128.

97. L.Y. Xiao, F.L. Jin, B.Z. Wang, Q.H. Liu, and W. Shao, IEEE Antennas Wirel. Propag. Lett. 9, 992 (2020).

98. A. Pitilakis, A. Tasolamprou, C. Liaskos, F. Liu, O. Tsilipakos, X. Wang, M. Mirmoosa, K. Kossifos, J. Georgiou, A. Pitsilides, N. Kantartzis, S. Ioannidis, E. Economou, M. Kafesaki, S. Tretyakov, and C. Soukoulis, in 12th International Congress on Artificial Materials for Novel Wave Phenomena (Metamaterials) (2018), p. 483.

Publisher's Note Springer Nature remains neutral with regard to jurisdictional claims in published maps and institutional affiliations. 